\title{
On the wake-induced vibration of tandem circular cylinders: the vortex interaction excitation mechanism
}

\author{
G. R. S. $A \operatorname{ASI}^{1} \dagger$, P. W. BEARMAN ${ }^{1} A N D$ J. R. MENEGHINI \\ ${ }^{1}$ Department of Aeronautics, Imperial College, London SW7 2AZ, UK \\ ${ }^{2}$ Department of Mechanical Engineering, NDF, POLI, University of São Paulo, \\ São Paulo, 05508-900, Brazil
}

(Received 28 October 2009; revised 25 May 2010; accepted 1 June 2010; first published online 16 August 2010)

The mechanism of wake-induced vibrations (WIV) of a pair of cylinders in a tandem arrangement is investigated by experiments. A typical WIV response is characterized by a build-up of amplitude persisting to high reduced velocities; this is different from a typical vortex-induced vibration (VIV) response, which occurs in a limited resonance range. We suggest that WIV of the downstream cylinder is excited by the unsteady vortex-structure interactions between the body and the upstream wake. Coherent vortices interfering with the downstream cylinder induce fluctuations in the fluid force that are not synchronized with the motion. A favourable phase lag between the displacement and the fluid force guarantees that a positive energy transfer from the flow to the structure sustains the oscillations. If the unsteady vortices are removed from the wake of the upstream body then WIV will not be excited. An experiment performed in a steady shear flow turned out to be central to the understanding of the origin of the fluid forces acting on the downstream cylinder.

Key words: flow-structure interactions, vortex flows, wakes/jets

\section{Introduction}

Great strides have been made in understanding the mechanisms involved in vortexinduced vibration (VIV) of an isolated circular cylinder free to vibrate transverse and/or in-line to a fluid flow. Progress has been reviewed by Sarpkaya $(1979,2004)$, Bearman (1984), Parkinson (1989), Blevins (1990) and Williamson \& Govardhan (2004). With the development of offshore oil fields and the deployment of riser pipes, much of the research related to cylinder response focused on conditions with low mass ratio $m^{*}$ and damping $\zeta$. Here, $m^{*}$ is the ratio of mass per unit length of the structure, $m$, to fluid displaced, $\rho \pi D^{2} / 4$, where $\rho$ is fluid density, $D$ is cylinder diameter and $\zeta$ is structural damping expressed as a fraction of critical damping. This research revealed a number of extremely interesting phenomena associated with VIV of isolated cylinders.

However, there are many flow-induced vibration (FIV) problems that involve two or more cylinders in configurations where the flow field of one cylinder influences

$\dagger$ Present address: Department of Naval Architecture and Ocean Engineering, NDF, POLI, University of São Paulo, Av. Prof Mello Moraes 2231, 05508-900, São Paulo SP, Brazil. Email address for correspondence: g.assi@usp.br 
the flow on one or more other cylinders. One of the best-known examples of closely spaced cylinder arrays that are susceptible to FIV is the crossflow heat exchanger. Other examples include arrays of offshore riser pipes, closely spaced chimneys and overhead conductor cables. If we now consider a cylinder array where the cylinders have low mass and damping then, compared to an isolated cylinder, the possibilities for response are greatly increased. In such a complex flow environment, one of the greatest challenges is to understand the physical mechanisms responsible for FIV.

We have approached this problem from a fundamental perspective by just considering two cylinders. An important advantage is that now the possible parameter space is much reduced and hence it should be easier to focus on some of the basic mechanisms responsible for FIV of cylinders in arrays. However, limiting the problem to two cylinders can still involve a large number of variables, hence we have imposed some further restrictions. The cylinders are of equal diameter, and when at rest, are aligned one behind the other in a tandem arrangement. Only the rear cylinder is free to respond and response is restricted to the direction transverse to the approaching stream. Also, we consider the cylinder to be rigid but flexibly mounted. Applying dimensional analysis to the primary variables, the response $\hat{y}$ is then given by the relation

$$
\frac{\hat{y}}{D}=\mathrm{f}\left(\frac{\rho U D}{\mu}, \frac{U}{D f_{0}}, \frac{x_{0}}{D}, m^{*}, \zeta\right),
$$

where $U$ is free-stream velocity, $\mu$ is viscosity, $f_{0}$ is the cylinder natural frequency and $x_{0}$ is the centre-to-centre spacing between the cylinders. It should be noted that $f_{0}$ is the natural frequency in vacuo and $U / D f_{0}$ is the reduced velocity. In practice, the cylinder frequency is measured in air but this provides a very close approximation to the natural frequency $f_{0}$.

A considerable amount of work has been published relating to the flow about two fixed cylinders in a tandem arrangement but substantially less has appeared on the response of tandem cylinders. Bokaian \& Geoola (1984) carried out experiments on tandem cylinders, to be described in more detail later, where the front one was fixed and the rear one was free to respond transverse to the flow. They observed that, depending on the value of $U / D f_{0}$, the response could be of the VIV type or it could be similar to galloping. They refer to the latter regime, which commences at values of reduced velocity beyond the peak response due to VIV, as wake-induced galloping.

Is this galloping similar to the classic kind described by den Hartog (1956) where a fluid-dynamic instability occurs, related to the cross-sectional shape of the body, such that motion of the body generates forces that increase the amplitude of vibration? It cannot be because when the rear cylinder is displaced in the transverse direction away from the line of centres, there is a hydrodynamic restoring force that is acting to return the cylinder to its original position. This suggests stability of the rear cylinder rather than instability. The restoring force was observed long before the work of Bokaian \& Geoola (1984) and prompted a number of researchers to develop theories to seek its origin and to predict its magnitude. Price (1976) concluded that none of the explanations that had been proposed were completely satisfactory.

Paidoussis \& Price (1988) developed mathematical models to describe the response of cylinders in closely spaced arrays and also simplified the problem by considering only two cylinders. They concluded that there needed to be a time delay between cylinder displacement and the transverse force to sustain wake-induced vibration (WIV). However, they did not provide a detailed explanation of the physical mechanism responsible for this delay beyond saying that there was likely to be a lag between the cylinder moving and the wake adjusting. Hence, two outstanding 


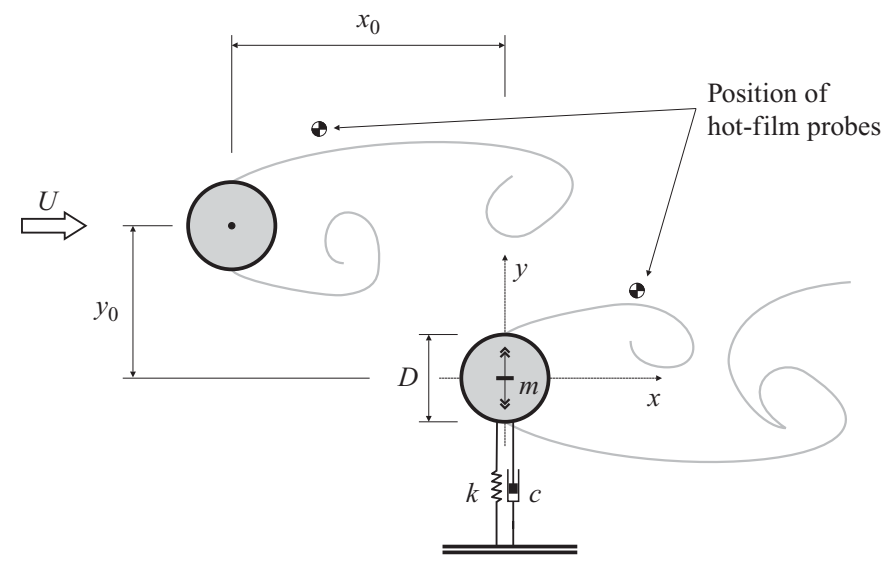

FIGURE 1. Arrangement of a pair of cylinders. The downstream cylinder is elastically mounted. The static upstream cylinder may be removed during experiments with a single cylinder.
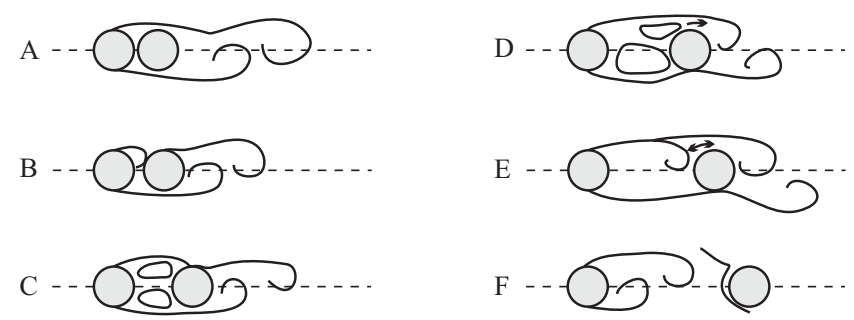

FIGURE 2. Regimes of flow interference for static cylinders for different tandem separations. Classification of interference regimes proposed by Igarashi (1981).

questions remain: what is the origin of the restoring force and what is the mechanism driving the transverse oscillation of the downstream cylinder in the regime described by Bokaian \& Geoola (1984) as wake-induced galloping?

\subsection{Flow interference around a pair of cylinders}

An arrangement of a pair of cylinders is shown in figure 1, and our interest is in the particular case where the line of centres is parallel to the free stream, i.e. $y_{0}=0$. The upstream cylinder is exposed to a free stream with velocity $U$, but the downstream body is immersed in a disturbed-flow region created by the wake of the upstream cylinder. The Reynolds number in this study is always based on the velocity approaching the upstream body. Vortices shed from the first body will not only pass by or impinge on the downstream cylinder, but will also interfere with vortex shedding from the downstream cylinder. Hence, if the downstream cylinder is mounted on an elastic base, the response of the body will be influenced by the wake coming from the upstream body.

Early experiments with tandem static cylinders identified two main interference regimes associated with the type of flow that develops in the gap between the bodies. The gap flow is characterized by the presence of two unstable shear layers that, depending on $x_{0}$ and $R e$, may reattach to the downstream cylinder, to form a region of recirculation, or roll up to initiate vortex shedding in the gap. Igarashi (1981) presented a more detailed classification dividing the gap-flow behaviour into six categories, as illustrated by ' $A$ ' to ' $F$ ' in figure 2. More recently, Sumner, Price \& 
Paidoussis (2000) produced a detailed classification of interference regimes for various configurations of staggered cylinders.

In this study, we are only concerned with the regime in which alternate vortex shedding is present in the gap. The transition from ' $\mathrm{E}$ ' to ' $\mathrm{F}$ ' in figure 2 marks the change to this type of flow. For a sufficiently large separation beyond a critical $x_{0}$, the shear layers start to roll up in the gap (pattern 'E'), and finally, a regime is reached in which a vortex street is formed behind the upstream cylinder (pattern ' $F$ '). Zdravkovich (1977) comments that the commencement of vortex shedding behind the upstream cylinder strongly affects and synchronises the vortex shedding behind the downstream one. Hence, both cylinders should be equally prone to flow-induced vibrations due to vortex shedding.' Other excitation mechanisms may occur for $x_{0}$ below the critical value, but the mechanism we are calling WIV only occurs when a developed wake is present in the gap.

The critical $x_{0}$ appears to have some dependency on Reynolds number and free-stream turbulence intensity. Ljungkrona, Norberg \& Sunden (1991) performed experiments around $R e=2.0 \times 10^{4}$ and observed that the critical separation is in the range $x_{0} / D=3.0-3.5$ for a low turbulence intensity of $0.1 \%$, while it decreases to $x_{0} / D=2.0-2.5$ for turbulence intensities of 1.4 and $3.2 \%$. Both Ljungkrona et al. (1991) and Zdravkovich (1986) suggested that this phenomenon may originate in instabilities in the separated shear layers from the upstream cylinder. Ljungkrona et al. (1991) also stated that the critical $x_{0}$ 'is high at very low $R e$, then decreases and passes through a minimum at moderate $R e$, followed by a maximum at higher $R e$ and then it begins to decay with increasing $R e$ '. They have found similarities between this behaviour and the $R e$ dependency of the vortex-formation length of single cylinders reported by Norberg \& Sunden (1987). Lin, Towfighi \& Rockwell (1995) and Norberg (1998) showed that it decreases with increases in $R e$ in the range $R e=10^{3}-10^{5}$, resulting in vortices forming closer to the base of the cylinder. Lin, Yang \& Rockwell (2002) and Assi (2005) also showed evidence of this phenomenon occurring with a pair of tandem cylinders.

Another less-pronounced variation in the critical $x_{0}$ may be due to hysteretic behaviour depending on whether cylinders are moved apart or brought closer together during the experiment. Liu \& Chen (2002) showed that this phenomenon occurs for a pair of square cylinders in tandem and Zdravkovich (1977) suggested this possibility may occur for circular cylinders. Additionally, numerical studies performed by Jester $\&$ Kallinderis (2003) for $R e=10^{3}$, Papaioannou et al. (2006) for $R e=10^{2}-10^{3}$ and Carmo, Meneghini \& Sherwin $(2010 a, b)$ for $R e=500$ also provide a clear demonstration of the hysteresis of both regimes in relation to $R e$ and $x_{0}$. All these aforementioned factors may cause the critical separation to present considerable variation.

The classification of flow interference regimes presented so far is for a pair of tandem cylinders that are stationary. Chen (1986) observed that 'When either or both of the cylinders are elastic or vibrate, the flow field becomes significantly more complicated because of the interaction of the fluid flow and the cylinder motion'. Assi et al. (2006) showed that when the downstream cylinder is allowed to oscillate, the interference between both bodies changes drastically depending on the amplitude of oscillation and Reynolds number.

\subsection{Wake-induced vibration of the downstream cylinder}

King \& Johns (1976) performed experiments in water $\left(R e=10^{3}\right.$ to $\left.2 \times 10^{4}\right)$ with two flexible cylinders for separations in the range $x_{0} / D=0.25-6.0$. They observed that for 
$x_{0} / D=5.5$, the upstream cylinder response showed a typical VIV pattern reaching amplitudes around $\hat{y} / D=0.45$ at the resonance peak, comparable to their tests with a single cylinder at the same $R e$. On the other hand, the downstream cylinder also started to build up oscillations together with the upstream one, but instead of the oscillations disappearing after the synchronization range, they remained at roughly the same level up to the highest reduced velocity tested. They classified the response of the downstream cylinder as a type of buffeting, since it originated from the wake interference coming from the upstream cylinder.

Brika \& Laneville (1999) performed tests with a pair of long cylinders in a wind tunnel in the range $R e=5000-27000$ with a flexible cylinder positioned from 7 to 25 diameters downstream of a rigid cylinder. They presented amplitude data for different separations revealing that as $x_{0}$ increases, the interference effect from the upstream wake is reduced until the response resembles that of a single cylinder without any (or with very little) interference. It is interesting to note that even between separations 16 and 25 they were still able to identify some change in the interference effect with the second cylinder positioned so far downstream. Because their experiments were performed in air, the mass ratio $\left(m^{*}=821\right)$ was two orders of magnitude higher than other experiments in water. Yet their damping parameter was extremely low, resulting in a combined mass damping of only $m^{*} \zeta=0.068$.

Moving from fully flexible to flexibly mounted rigid cylinders, we refer to experiments performed by Zdravkovich (1985) with two rigid cylinders free to respond in 2 degrees of freedom (d.o.f.) mounted in a wind tunnel $\left(R e=1.5 \times 10^{4}\right.$ $9.5 \times 10^{4}, m^{*}=725$ and $\zeta=0.07$ ). Due to a very high combined mass-damping parameter $\left(m^{*} \zeta=50\right)$, Zdravkovich was only able to observe a build-up of oscillations for $x_{0} / D=4.0$ at reduced velocities beyond $U / D f_{0}=50$, asymptotically reaching a maximum of $\hat{y} / D=1.7$ at around a reduced velocity of 80 . Zdravkovich \& Medeiros (1991) performed similar 2-d.o.f. tests in a wind tunnel varying $m^{*} \zeta$ between 6 and 200 $\left(R e=5 \times 10^{3}-1.4 \times 10^{5}\right)$. A similar asymptotic behaviour for crossflow vibrations was observed for high reduced velocities. Their results revealed a strong dependency of the response on $m^{*} \zeta$ and showed that very high values of mass damping are required to inhibit WIV on the downstream cylinder. The maximum amplitude was obtained at their highest reduced velocity of 120 .

Moving a step further in the simplification of the problem, we find a few studies for rigid cylinders responding only in 1-d.o.f. Bokaian \& Geoola (1984) performed experiments with two cylinders in tandem in a water channel $(R e=700-2000)$. The upstream cylinder was fixed, while the downstream cylinder was elastically mounted on air bearings and free to respond only in the crossflow direction. They varied centreto-centre separation in the range $x_{0} / D=1.09-5.0$ covering the interference regimes with and without vortex shedding in the gap. Results for amplitude of response versus reduced velocity (with $f_{W}$ being the natural frequency in still water) are presented in figure 3(a) for three values of $x_{0}$. A vigorous build-up of oscillations with increasing flow speed was observed for all flow speeds greater than a critical threshold velocity. Such a severe 1-d.o.f. vibration was observed to resemble the response due to classical galloping of non-circular bodies and they referred to the phenomenon as 'wake-induced galloping'. Bokaian \& Geoola (1984) concluded that depending on $x_{0}, m^{*}$ and $\zeta$, the downstream cylinder 'exhibited vortex-resonance, or galloping, or a combined vortex-resonance and galloping, or a separated vortex-resonance and galloping' response.

In figure 3(a), two examples of these different responses are found, with $x_{0} / D=1.5$ presenting a vortex resonance that is followed by (or combined with) 

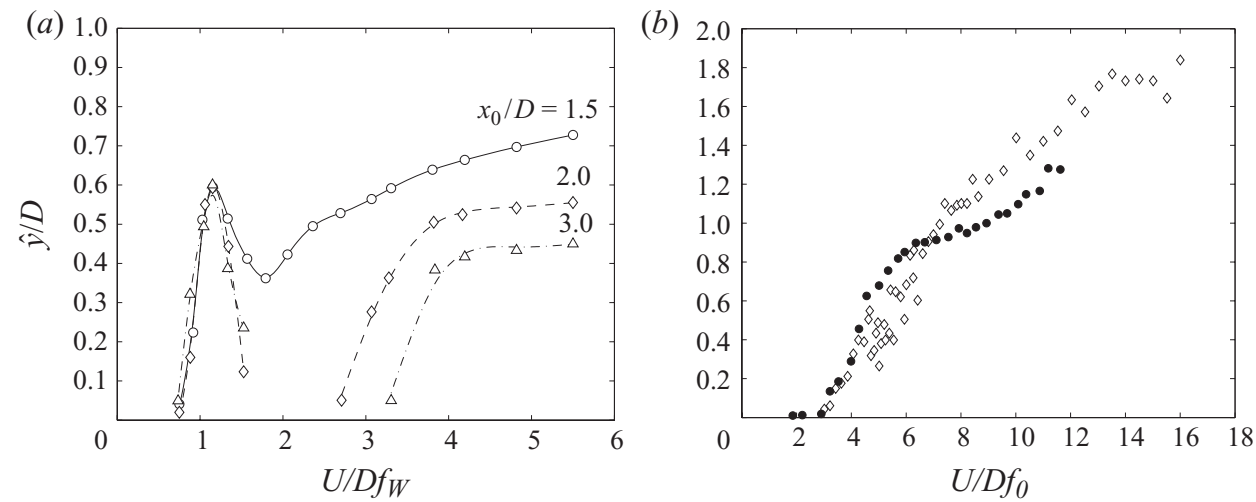

FiguRE 3. WIV response of the downstream cylinder. (a) Varying $x_{0}, m^{*}=8.4, \zeta=0.013$ and $R e=700-2000$ (Bokaian \& Geoola 1984). (b) $\diamond, x_{0} / D=4.75, m^{*}=3.0, \zeta=0.04$ and $R e=3 \times 10^{4}$ (Hover \& Triantafyllou 2001); $\bullet, x_{0} / D=4.0, m^{*}=1.9, \zeta=0.007$ and $R e=3000-13000$ (Assi et al. 2006).

a 'galloping response', and $x_{0} / D=2.0$ and 3.0 presenting separated vortex-resonance and 'galloping' regimes. Unfortunately, the form of the flow in the gap is not known; however, from the classification of regimes proposed by Igarashi (1981) for static cylinders, and shown in figure 2, it seems likely that for the smallest gaps vortex shedding did not occur ahead of the downstream cylinder. Hence, it is not apparent if the 'galloping-type' response found by Bokaian \& Geoola (1984), for all the separations shown in figure $3(a)$, is the WIV that we are studying. A mechanism of gap-flow switching sustains vibrations of cylinders in close proximity, as described by Zdravkovich $(1974,1988)$. It occurs for the first regime of flow interference when the upstream shear layers reattach to the downstream cylinder and no vortex wake is developed in the gap flow. Zdravkovich (1974) offers a convincing explanation for the excitation of tandem cylinders in close proximity, but leaves open the question of the mechanism when cylinders are farther apart.

A clearer example of WIV is shown in the work of Hover \& Triantafyllou (2001) who measured displacements and forces for rigid cylinders in a water-towing tank at a constant $R e=3 \times 10^{4}$. They used a closed-loop control system that forces the oscillation of a cylinder in response to the fluid force measured on the cylinder. Using this technique, they are able to control the key parameters: $f_{0}, m^{*}$ and $\zeta$. Their constant $R e$ results presented in figure $3(b)$ were obtained by keeping $U$ constant and varying $f_{0}$. For their experiments, $m^{*} \zeta=0.12$, which is very close to the value of $m^{*} \zeta=0.11$ obtained by Bokaian \& Geoola (1984). The differences in the levels of amplitude found in the two investigations is probably related to a difference of one order of magnitude in $R e$, as discussed in Assi (2009). For a separation of $x_{0} / D=4.75$, Hover \& Triantafyllou (2001) observed one single branch of response that builds up monotonically reaching amplitudes of $[\hat{y} / D]_{\max }=1.9$ for reduced velocities around 17.

More recently, Assi et al. (2006) performed 1-d.o.f. experiments with two rigid cylinders in a recirculating water channel $\left(R e=3 \times 10^{3}-1.3 \times 10^{4}\right)$. Their measurements of the transverse amplitude of the rear cylinder, also presented in figure $3(b)$, were obtained at $R e$ values close to those tested in the experiments carried out by Hover \& Triantafyllou (2001). However, Assi et al. (2006) employed a very low damping elastic system resulting in $m^{*} \zeta=0.013$, which is one order of magnitude lower. 
Nevertheless, both sets of results are in reasonably good agreement showing the expected WIV branch of high-amplitude oscillation building up as reduced velocity is increased. However, the results of Assi et al. (2006) also show a smooth hump around $U / D f_{0}=6.0$, indicating a VIV response.

\subsection{The wake-induced vibration mechanism}

A major difficulty in using quasi-steady arguments to explain the origin of WIV is that when the rear cylinder of a tandem pair is displaced sideways, a force develops that is acting to return the cylinder to its original position. The various theories about how this force is generated have been well explained by Price (1976) and he concludes that none can successfully predict the magnitude of the force.

Zdravkovich (1977) proposed that the displacement of the wake of the upstream cylinder by the downstream one towards the wake centreline would induce a lift force towards the centreline. Maekawa (1964) attributed the origin of the force to a buoyancy effect. The static pressure is a minimum at the centreline of the wake; hence, the pressure gradient across the wake generates a buoyancy force towards the centreline. However, Best \& Cook (1967) and Wardlaw \& Watts (1974) showed, by integrating the pressure field around the downstream cylinder, that only $30-50 \%$ of the total lift could be attributed to a buoyancy effect. Maekawa (1964) also suggested that turbulence generated by the wake may affect the separation on the internal side of the cylinder, changing the symmetry of the pressure field around the downstream body. However, this phenomenon was not confirmed by experiments that examined transition on the downstream cylinder.

Mair \& Maull (1971) proposed that the side force was caused by an entrainment of flow into the wake of the upstream cylinder generating an inclined free-stream velocity approaching the downstream body. The lift force towards the centreline would then be due to resolved drag, i.e. the component of inclined drag that contributes in the crossflow direction of displacement. Using estimated flow inclination angles, Price (1976) estimated that the lift force due to resolved drag is only $25 \%$ of the total lift-force measure on the cylinder. Rawlins, referenced in Price (1976), stated that because of variations of velocity across the wake, the boundary layer on the downstream cylinder feeds different amounts of vorticity into the associated shear layers. He concluded that a circulation is built up around the cylinder until rates at which vorticity is discharged into the two separated shear layers are equal. This circulation would generate lift towards the centreline. Rawlins' lift curves have the same general shape as the lift profile measured experimentally, but do not obtain the required magnitude, generating only $75 \%$ of the lift measured at the position of the maximum. Hence, following the detailed study by Price (1976) of the available theories, it appears that there is no fully satisfactory explanation for the origin of the transverse force acting on the rear cylinder of a cylinder pair.

It is clear that a simple quasi-steady theory will not be able to predict the fluidelastic instability of the downstream cylinder if it is only free to oscillate in 1-d.o.f. Price (1984) improved a quasi-steady model by inserting a time delay between the cylinder displacement and the fluid force. This phase lag was intended to account for a possible flow retardation generated in the gap flow between the pair of cylinders; however, the physical origin of this effect is unclear. Granger \& Paidoussis (1996) proposed yet another improvement of the quasi-steady theory employed by Price (1984) with the aim of modelling the most relevant unsteady effects neglected by the quasi-steady approach. In essence, their model, referred to as quasi-unsteady, incorporates a memory effect into the same time-delay idea, "the physical origin 
of which arises from the diffusion-convection process of the vorticity induced by successive changes in the velocity of the body'.

Paidoussis, Mavriplis \& Price (1984) employed potential-flow theory to investigate fluid-elastic instability of an array of cylinders. Although they were not calculating any viscous forces in their model itself, they had to include a phase-lag effect in the fluid force in order to generate any oscillatory instability. Probably, the most intuitive explanation for the existence of a phase lag on the fluid force was offered by Paidoussis \& Price (1988), who attributed this effect to a time delay associated with the reorganization of the viscous wake flow as the cylinder is displaced. Paidoussis et al. (1984) concluded that if viscous effects are neglected altogether, then the only form of instability possible is divergence, which is a static, non-oscillatory instability'.

Each improved quasi-steady model gave better agreement with experimental data and they all include a phase lag between the cylinder displacement and the fluid force, yet a precise explanation for the origin of this time delay has not been produced. In this paper, we aim to study the mechanisms responsible for WIV of the downstream cylinder of a tandem pair.

\section{Experimental set-up}

Experiments were performed in the Department of Aeronautics at Imperial College London. Tests were carried out in a recirculating water channel with a free surface and a test section $0.6 \mathrm{~m}$ wide, $0.7 \mathrm{~m}$ deep and $8.0 \mathrm{~m}$ long. The side walls and bottom of the section were made of glass mounted on a steel frame, allowing a complete view of the models for flow-visualization purposes. Flow speed was continuously variable and an electromagnetic flow meter provided a reading of the instantaneous flow rate, which was divided by the area of the test section yielding the value of $U$. Free-stream turbulence intensity mapped across the section was $(3.1 \pm 0.7) \%$ on average. Bokaian \& Geoola (1984) performed experiments with a pair of cylinders in a water channel and found no significant difference in their WIV results for freestream turbulence intensity of 6.5 and $11.9 \%$. The actual flow quality proved to be adequate to perform our FIV study. This was validated by good agreement between our preliminary VIV results for an isolated cylinder and other experiments presented in the literature (as will be shown later).

Two circular cylinders were made from a $50 \mathrm{~mm}$ diameter acrylic tube, giving a maximum $R e=30000$, based on cylinder diameter $D$. With a wetted length of $650 \mathrm{~mm}$ (total length below water level), the resulting aspect ratio of the model was 13 . The cylinders were hollow and contained air in order to keep the mass low. It was judged preferable not to install end plates on the cylinder in order not to increase the fluid damping in the system. Instead, it was chosen to have the models terminating as close as possible to the glass floor of the test section. One single cylinder occupies $8.3 \%$ of the total area of the test section. If one cylinder is oscillating behind the other, the maximum projected area of both cylinders would result in a blockage ratio of $16.6 \%$ if it is displaced by more than $1 D$. Brankovic (2004) performed VIV tests on a single cylinder in the same water channel with three ratios of blockage: $11.3,13.6$ and $17 \%$. She concluded that although the maximum amplitude of oscillation decreased slightly for higher blockage ratios, the results remained qualitatively the same, meaning that the hydrodynamic mechanism did not change for the three cases studied.

The upstream cylinder was rigidly attached to the structure of the channel preventing displacements in any direction, while the downstream cylinder was fixed at its upper end to a 1-d.o.f. elastic mounting. The initial streamwise and crossflow 


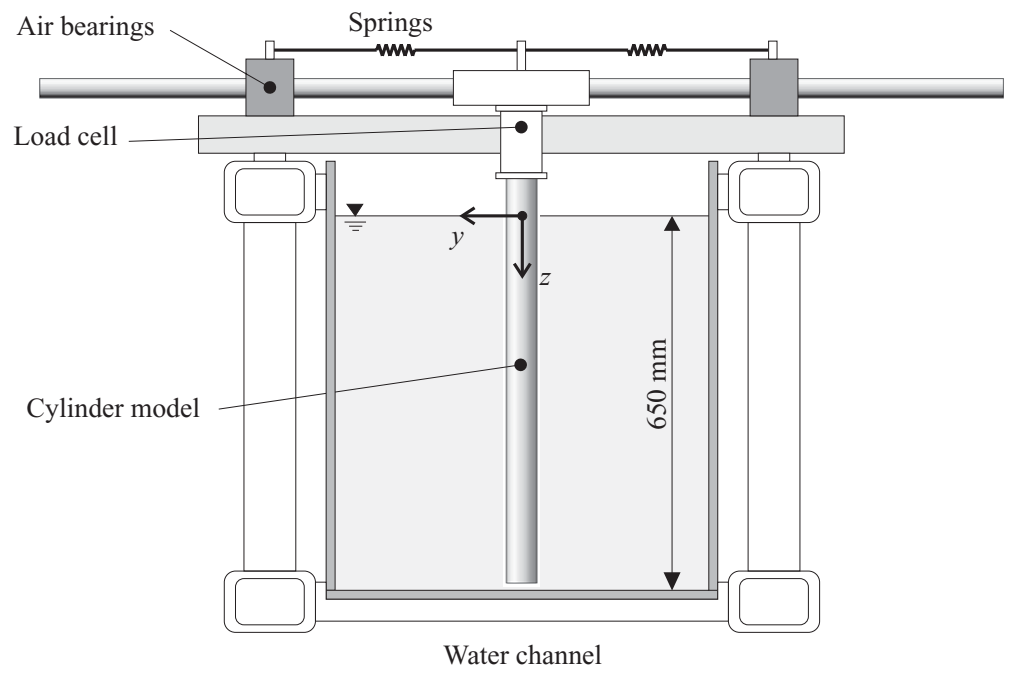

FIGURE 4. Schematic representation of the 1-d.o.f. rig holding the downstream cylinder.

The free stream flows out of the page in the $x$-axis direction.

separations between cylinders ( $x_{0}$ and $y_{0}$ in figure 1 ) could be varied by changing the position of the upstream model, so that the downstream cylinder always oscillated around the centreline of the test section. For most of the experiments performed in this study, both cylinders were initially aligned with the free-stream direction $\left(y_{0}=0\right)$, arranged in a tandem configuration. Figure 4 shows a schematic representation of the 1-d.o.f. rig on which the downstream cylinder was mounted. The models were aligned in the vertical direction passing through the free-water surface and the downstream cylinder was mounted such that there was a $2 \mathrm{~mm}$ gap between the lower end of the cylinder and the glass floor of the test section. The support system was firmly attached to the channel structure and sliding cylindrical guides were free to move in the transverse direction defined as the $y$-axis. A pair of coil springs connecting the moving base to the fixed supports provided the restoration force for the system. A pair of sliding guides made out of a carbon fibre tube with a smooth finish ran through air bearings at each side. The tubes were connected at mid-length by a light, stiff platform machined out of a block of aluminium, to which the cylinder was firmly attached.

It is known that the dynamic response of a cylinder is extremely sensitive to the structural characteristics of the system; therefore, extra care was taken to determine the precise value of natural frequency, mass and damping of the structure. The spring stiffness $(k)$ combined with the mass of all oscillating parts $(m)$ resulted in a natural frequency of oscillation of $f_{0}=0.30 \mathrm{~Hz}$, determined by performing a series of free decay tests in air. It was possible to vary reduced velocity to a maximum of $U / D f_{0}=40$ when $U$ was increased up to $0.6 \mathrm{~m} \mathrm{~s}^{-1}$. The minimum flow speed in the channel was $U=0.03 \mathrm{~m} \mathrm{~s}^{-1}$, resulting in the lower limit of $U / D f_{0}=2.0$. All moving parts of the elastic base contributed to the effective mass oscillating with the cylinder, resulting in a mass ratio of $m^{*}=2.6$ (calculated as the total mass divided by the mass of displaced water). The air bearings proved to be an effective way to reduce damping without compromising the stiffness of the structure, especially in resisting drag loads for higher flow speeds. By carrying out free decay tests in air, it was also possible to estimate the structural damping of the system resulting in $\zeta=0.7 \%$, calculated 
as a percentage of the critical damping. Therefore, the product $m^{*} \zeta=0.018$ for the majority of the experiments.

A load cell was attached between the model and the platform to measure instantaneous and time-averaged hydrodynamic forces acting on the cylinder. It consisted of two independent load cells machined out of one block of a hard aluminium alloy. The cells were perpendicular to each other in order to measure components of the hydrodynamic force in the streamwise and crossflow directions lift and drag, respectively. Each cell was individually calibrated up to $50 \mathrm{~N}$ with an uncertainty of $1 \%$ and no significant cross-talk between them was observed. Drag could be measured directly by the load cell, since the cylinder was not allowed to move in the $x$-axis. However, the crossflow component needed to be corrected in order to remove the inertia force due to the moving mass of the cylinder.

An optical positioning sensor was installed to measure the $y$-displacement of the cylinder without affecting the damping. Completing the instrumentation, a pair of hot-film probes was employed to measure velocity fluctuations in the gap between the cylinders and in the developed wake downstream of the second cylinder (see figure 1). A Dantec particle-image velocimetry (PIV) system was used to map velocity fields. A laser sheet entered the section through one of the side walls illuminating the flow at the mid-height of the section. A digital camera was positioned underneath the channel and the illuminated plane was visualized through the glass floor. Flow visualization was also carried out using the same laser to illuminate fluorescent dye or hydrogen bubbles. More details about the experimental set-up, flow quality, the design of the load cell and operation of the 1-d.o.f. rig can be found in Assi (2009).

\section{Analytical modelling and preliminary results}

A preliminary experiment was performed with a single cylinder free to oscillate in 1-d.o.f. in a uniform flow to serve as validation of the experimental methodology. The analytical modelling as well as the VIV results that follows will be useful when understanding the WIV excitation mechanism.

Allowing for displacements only in 1-d.o.f., the equation of motion for an elastically mounted body is expressed by

$$
m \ddot{y}+c \dot{y}+k y=\frac{1}{2} \rho U^{2} D L\left[\bar{C}_{y}+\hat{C}_{y} \sin (2 \pi f t+\phi)\right],
$$

where $y, \dot{y}$ and $\ddot{y}$ are, respectively, the displacement, velocity and acceleration of the body and $C_{y}(t)$ is the time-dependent fluid force coefficient in the crossflow direction (lift). Following an analysis presented by Parkinson (1971) and others, the displacement of a cylinder under VIV may be expressed by the harmonic response

$$
y(t)=\hat{y} \sin (2 \pi f t),
$$

where $\hat{y}$ and $f$ represent the harmonic amplitude and frequency of oscillation, respectively. For large-amplitude oscillation under a steady-state regime of VIV, the fluid force and the body response oscillate at the same frequency $f$, which is usually close to the natural frequency of the system. According to this 'harmonic forcing and harmonic motion' assumption $C_{y}$ can be expressed by a time-average term $\bar{C}_{y}$ and a transient term modelled as a sine wave with amplitude $\hat{C}_{y}$, frequency $f$ and phase delay between the transverse force and the motion of $\phi$. In our single-cylinder experiment, we expect $\bar{C}_{y}$ to be 0 .

Throughout this study, cylinder-displacement amplitude $(\hat{y} / D)$ was found by measuring the root-mean-square value of response and multiplying by $\sqrt{2}$. Such 

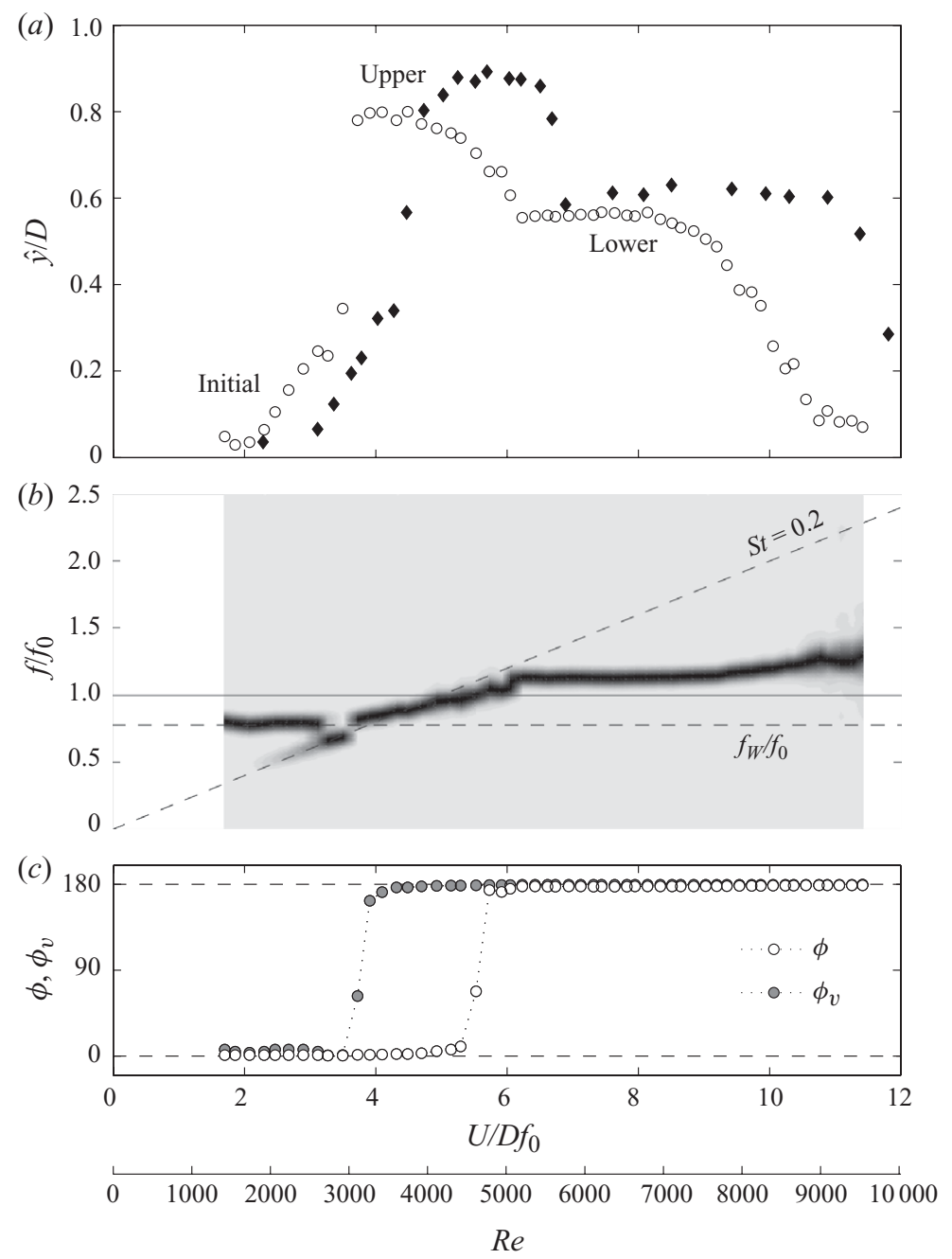

FiguRE 5. VIV response of a single cylinder free to oscillate in the crossflow direction. (a) Displacement ( $O$, the present study; $\bullet$ Khalak \& Williamson $(1997), m^{*}=2.4, \zeta=0.0059$ ). (b) Normalized PSD of frequency of oscillation. (c) Phase angle between lift and displacement.

a harmonic amplitude assumption is likely to underestimate the maximum response but was judged to be acceptable for assessing the average amplitude of response for many cycles of steady-state oscillations. An alternative method was to measure the amplitude of individual peaks of displacement in order to estimate an average value, but this was found to give very similar results to the harmonic amplitude mentioned above, thus proving that a sinusoidal approximation for the response is indeed very reasonable. The same procedure was employed to determine the magnitude of all other fluctuating variables, such as $\hat{C}_{y}$ and $\hat{C}_{x}$.

Figure 5 presents the response of the single cylinder under VIV. Since $U$ is increased in order to vary the reduced velocity, $R e$ also varies along the reduced-velocity axis and is plotted as a reference in a parallel axis. In the $\hat{y} / D$ curve, it is possible to identify the typical three branches of response for low $m^{*} \zeta$ systems discussed by Williamson \& Govardhan (2004) - initial, upper and lower branches - clearly 
defining a finite synchronization range. In the original curve presented by Khalak \& Williamson (1997), the reduced velocity was calculated by employing the natural frequency measured in still water $\left(U / D f_{W}\right.$, in our notation); hence, their curve plotted here has been offset in order to agree with our $U / D f_{0}$ axis (considering an added mass coefficient of $C_{a}=1.0$ ). In the $f / f_{0}$ graph, a variation from light to dark grey represents dominant peaks in the normalized power spectral density (PSD) of the frequency of oscillation (see Assi (2009) for more details).

As discussed by Williamson \& Govardhan (2004), the total fluid force acting on the cylinder can be divided into two components: a potential-force component $C_{y_{P}}$, given by the ideal flow inertia force, and a vortex-force component $C_{y_{v}}$, due only to the dynamics of the vorticity field around the body. By definition, $C_{y_{P}}$ is always opposing the body's acceleration and its magnitude is proportional to the product of the displaced fluid mass and the acceleration of the body. On the other hand, $C_{y_{V}}$ essentially depends on the dynamic of vortices in the wake and may be expressed in terms of another $\hat{C}_{y_{V}}$ and phase angle $\phi_{V}$ in relation to the displacement of the cylinder, resulting in

$$
\hat{C}_{y} \sin (2 \pi f t+\phi)=\hat{C}_{y_{P}} \sin \left(2 \pi f t+180^{\circ}\right)+\hat{C}_{y_{V}} \sin \left(2 \pi f t+\phi_{V}\right) .
$$

This decomposition is useful when analysing the actual contribution the vortices in the wake are having on the total force acting on the cylinder. The almost $180^{\circ}$ phase shift experienced by $\phi$ and $\phi_{V}$, associated with the transition in the vortex-shedding mode, is also clearly identified in figure 5(c). Khalak \& Williamson (1999) verified that changes in $\phi$ and $\phi_{V}$ are related to changes in the modes of vortex shedding.

A careful analysis of the time series will serve as reference for the discussion of WIV results to come later. Time series of $\hat{y} / D$ are plotted for different reduced velocities in figure $6(a, c, e)$ (where $T$ is the period of an average cycle). The first data set $\left(U / D f_{0}=4.0\right)$ is collected from a point in the upper branch of VIV; the second $\left(U / D f_{0}=5.7\right)$ is in the transition from the upper to the lower branch; and the third $\left(U / D f_{0}=7.9\right)$ is in the lower branch. In the first and third series, it is possible to note that the envelope of $\hat{y} / D$ is more regular than during the transition between branches.

The plots in figure $6(b, d, f)$ compare several cycles of $\hat{y} / D$ and $C_{y}$ superimposed in one figure, each representing around $20 \%$ of the total number of acquired cycles with displacement around the average $\hat{y} / D$. Again, it is evident that the deviation of $\hat{y} / D$ from the mean curve (thick line in black) is accentuated during the transition between branches. Looking at $C_{y}$ curves of figure 6 , we observe that although the magnitude of lift shows considerable variations, the phase angle between cycles is reasonably constant in the upper and lower branches. $C_{y}$ is clearly almost in phase with $\hat{y}$ in the upper branch and out of phase in the lower. However, the behaviour of $C_{y}$ for only a few cycles of oscillation is enough to show that a constant phase angle is not observed during the transition from the upper to the lower branch. This is the intermittence phenomenon described by Khalak \& Williamson (1999), and it is clear from the graph that both magnitude and phase of the lift are changing during the transition between modes.

$\hat{C}_{y}$ was directly measured with the load cell, and $\hat{C}_{y_{p}}$ and $\hat{C}_{y_{V}}$ were calculated as suggested by Williamson \& Govardhan (2004). Force measurements and decomposition were in good agreement with results presented by Khalak \& Williamson (1999), but are not presented here for brevity. In addition, PIV measurements also found good agreement with their work in identifying the 

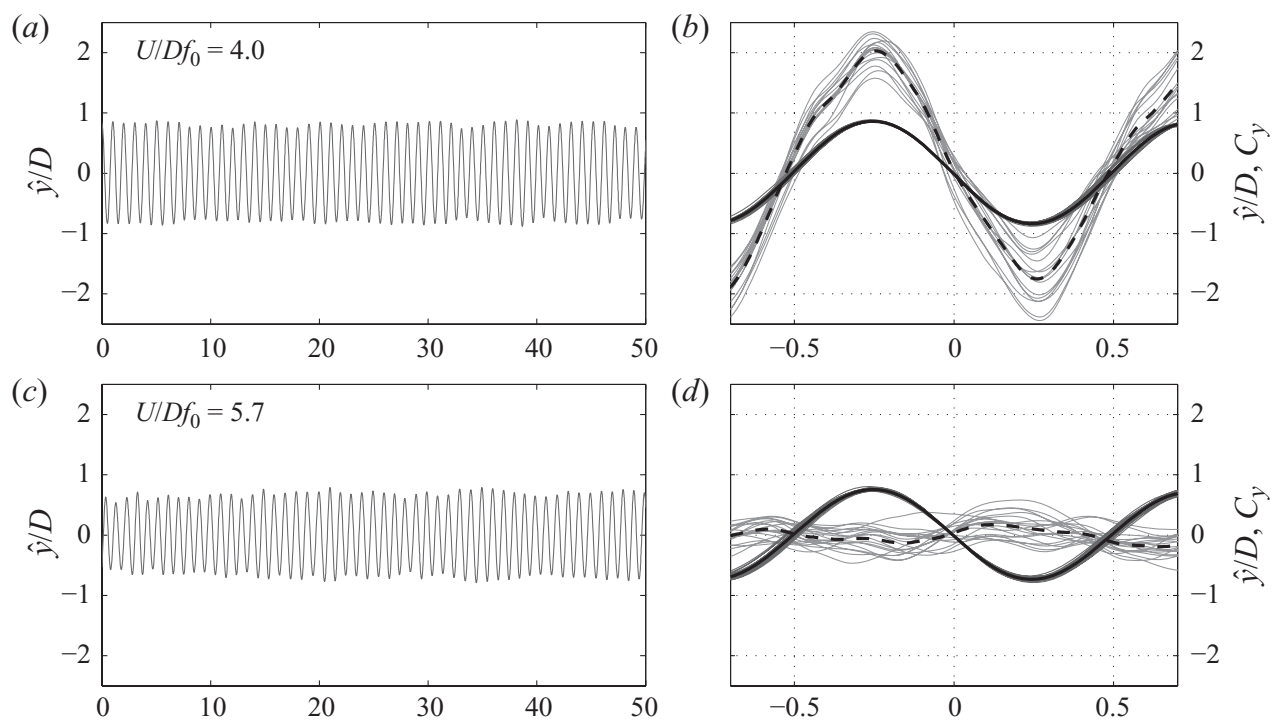

(d)
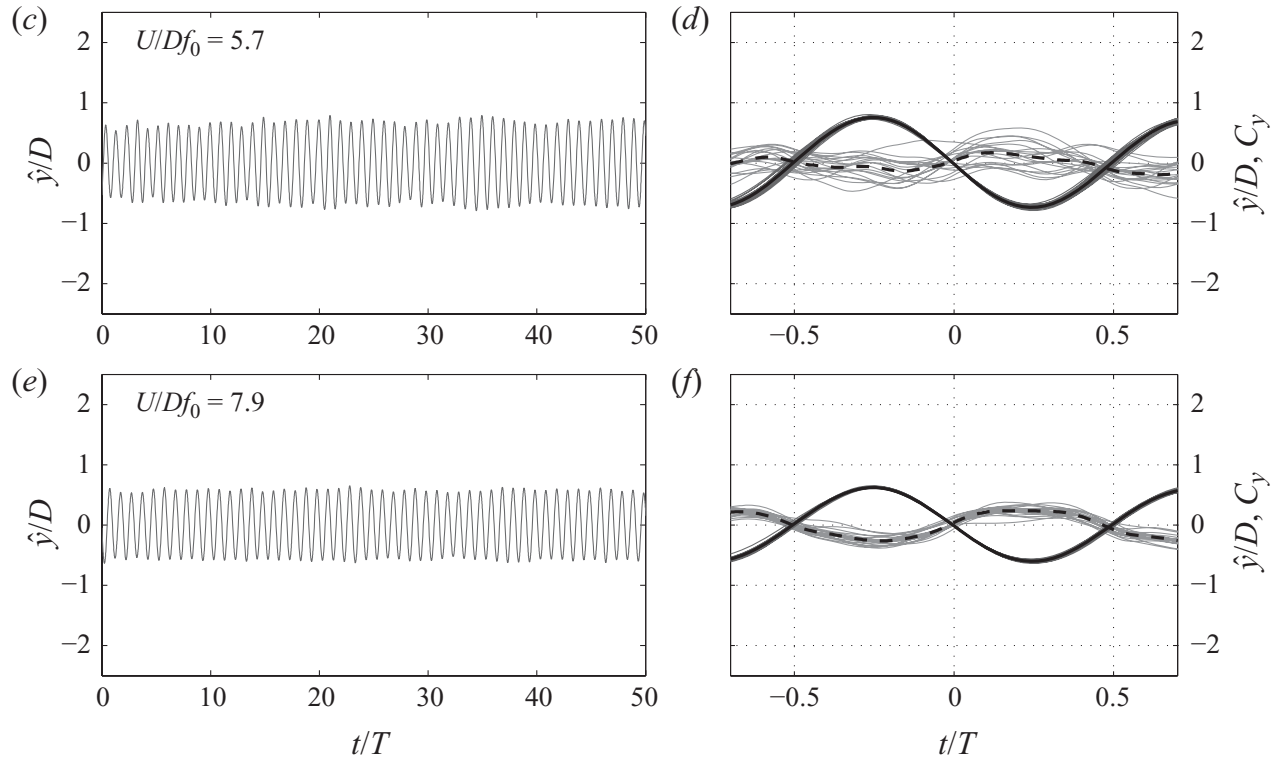

Figure 6. $(a, c, e)$ Time series of displacement for around 50 cycles of oscillation. $(b, d, f)$ Superimposed plots of similar cycles. $\hat{y} / D$ in dark grey and $C_{y}$ in light grey with average cycle in black.

appropriate vortex-shedding modes in the wake. These results can be found in Assi (2009).

\section{WIV of the downstream cylinder}

We have investigated WIV responses for the downstream positioned at various locations beyond the critical separation of $x_{0} / D=2.5$ and the results are presented in figure 7. The upstream cylinder was stationary and only the downstream cylinder was free to respond in 1-d.o.f. in the crossflow direction ( $y$-axis). Fully developed vortices were observed in the gap flow for all separations with $x_{0} / D>2.5$.

The response curves demonstrate that the overall amplitude of vibration in the WIV regime decreases as the cylinders are moved farther apart. The smallest separation of $x_{0} / D=4.0$ presented the highest amplitudes of vibration with increasing amplitude for higher reduced velocities. In contrast, for $x_{0} / D=20.0$, the response is drastically reduced and, overall, more closely resembles that of VIV of a single cylinder. As will be explained later, the WIV mechanism that excites high-amplitude vibrations grows weaker as the cylinders are separated, until the interference is irrelevant and the cylinder behaves like an isolated body. 


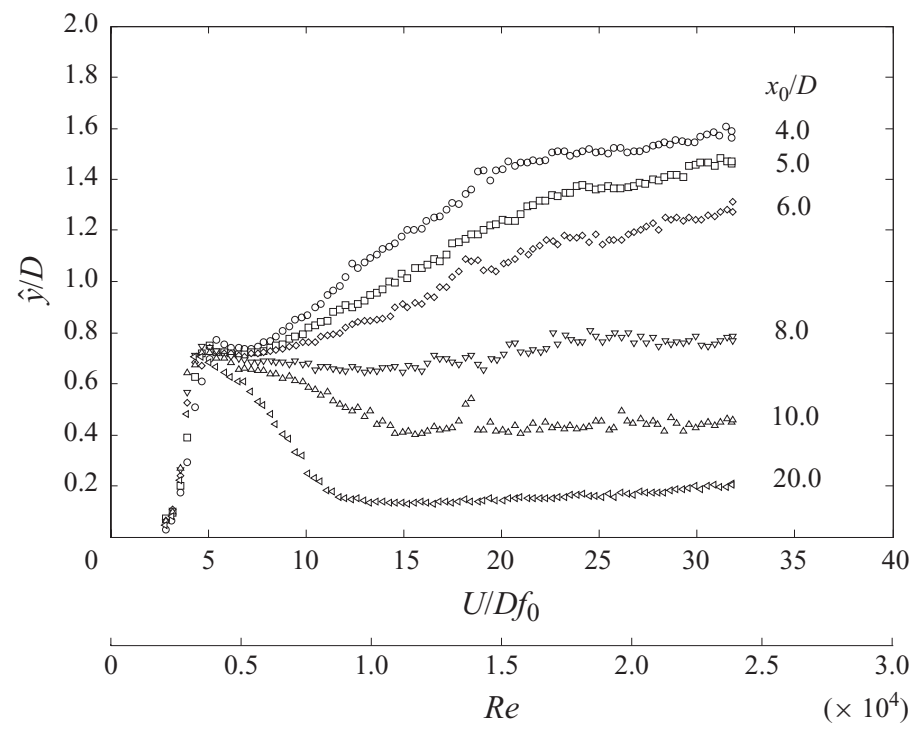

FIGURE 7. WIV response of the downstream cylinder at various $x_{0} / D$ separations.

In order to develop a clearer understanding of the WIV excitation mechanism, we will focus our attention on a single separation of $x_{0} / D=4.0$, as will be discussed in detail in the following sections.

\subsection{WIV of the downstream cylinder at $x_{0} / D=4.0$}

A separation of $x_{0} / D=4.0$ was chosen for various reasons: (i) it was beyond the critical separation where a bistable reattachment of the shear layers may occur and vortex shedding was observed to be present in the gap for all flow speeds; (ii) it gives a WIV response that is qualitatively consistent with that for larger separations; (iii) the displacements and magnitudes of fluid forces were comparatively large and can be measured to a good accuracy; (iv) it was sufficiently small to allow the wakes of both cylinders to be measured simultaneously using PIV.

Figure $8(a)$ plots the displacement versus reduced velocity. $\hat{y} / D$ is the harmonic amplitude of displacement discussed above and gives a good measure of the average amplitude of vibration for many cycles of oscillation. However, $\hat{y} / D$ does not provide a good estimate of the maximum amplitude that the cylinder might reach if displacement varies from cycle to cycle. By considering individual peaks of oscillation, it was possible to estimate a maximum and a minimum peak amplitude taking an average of the $10 \%$ highest and lowest peaks of the whole series, yielding $[\hat{y} / D]_{\max }$ and $[\hat{y} / D]_{\min }$, respectively. Therefore, we can say that for a certain reduced velocity, the cylinder oscillates on average with $\hat{y} / D$, but reaches the maximum and minimum limits given by the other curves. This provides considerable new information about the response since it shows that $\hat{y} / D$ is not only building up with reduced velocity, but also the deviation from the average amplitude, i.e. the irregularity of the envelope, is increasing.

Figure $8(b)$ shows that $f$ increases above $f_{0}$ but does not reach the line for $S t=0.2$. The PSD contours reveal that for any other frequencies present in the spectrum, the levels are much smaller than the dominant branch that is evident across the reducedvelocity range. That is to say, there is no significant trace of a frequency branch 

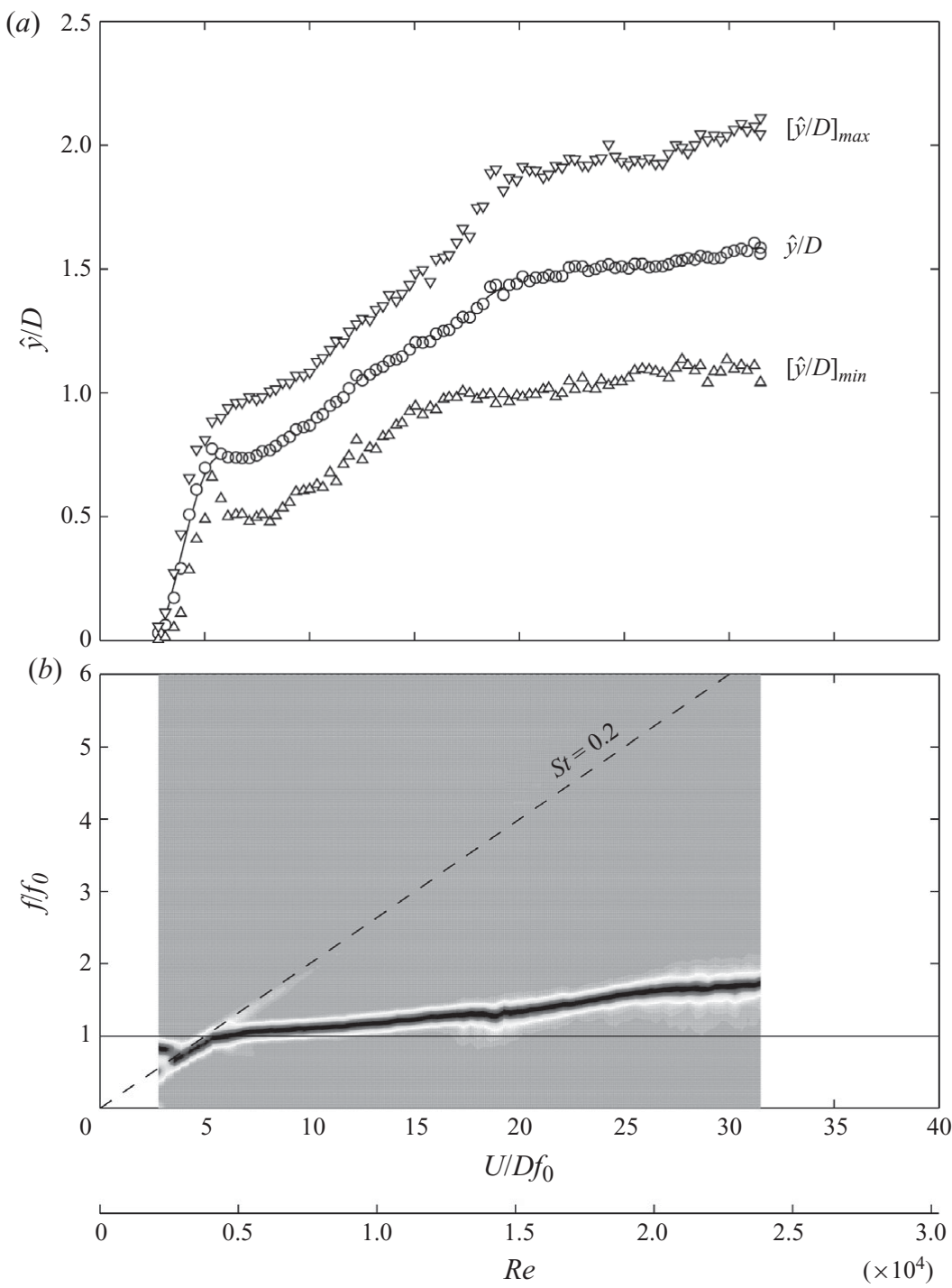

FIGURE 8. WIV response of the downstream cylinder at $x_{0} / D=4.0$. (a) Displacement; (b) normalized PSD of frequency of oscillation.

associated with $S t=0.2$ beyond reduced velocity of 10 , with only a hint appearing between 5 and 10 .

As shown in figure 6, the envelope of the single-cylinder VIV response is fairly regular except during the transition between branches. This is generally not the case for the WIV response, as illustrated in figure 9. We note that the envelope of response is already irregular at $U / D f_{0}=4.6$ and becomes more irregular for higher reduced velocities. This is also revealed in the plots shown in figure $9(b, d, f)$, which show the cycles for the $20 \%$ highest peaks.

By comparing the variation of $C_{y}$ and $\hat{y} / D$ in one cycle, it is possible to estimate the phase between them and also the overall frequency content of the signals. For $U / D f_{0}=4.6$, very close to the VIV resonance region, we note that lift and displacement 

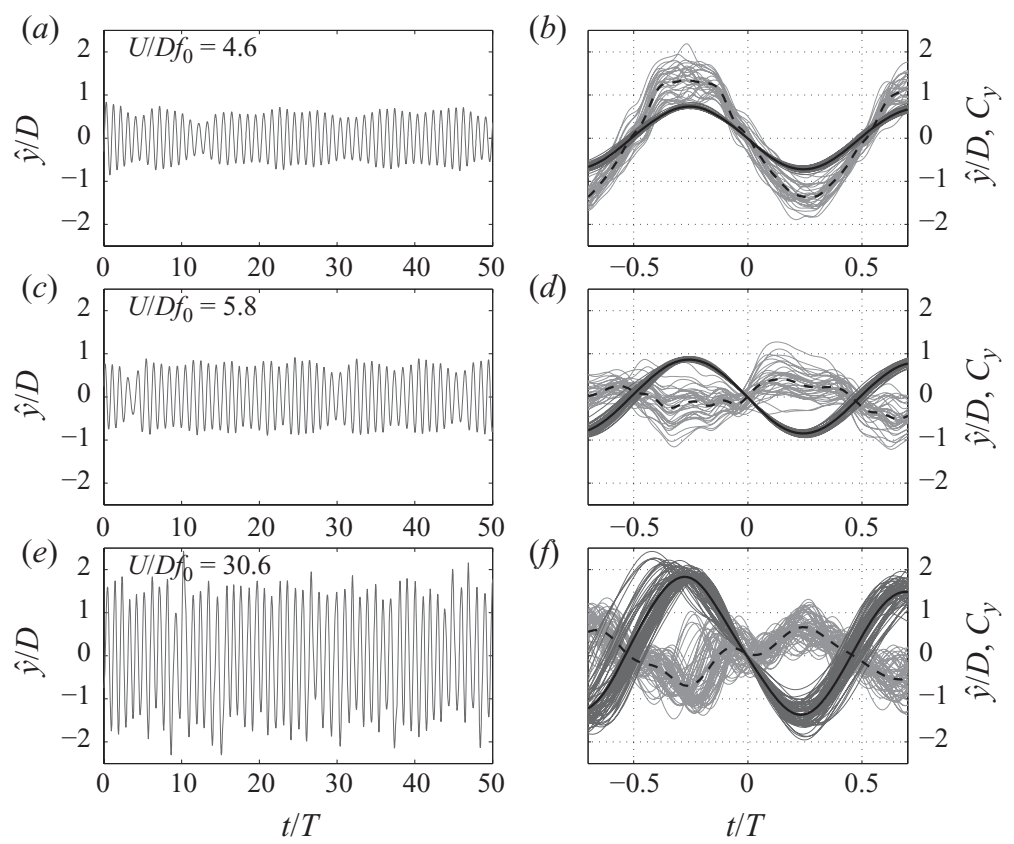

FIGURE 9. $(a, c, e)$ Displacement signal for around 50 cycles of oscillation. $(b, d, f)$ Superimposed plots of similar cycles; $\hat{y} / D$ in dark grey and $C_{y}$ in light grey with average cycle in black.

are almost in phase and there seems to be a single dominant frequency present in $C_{y}$. However, moving to a slightly higher reduced velocity of 5.8 , the behaviour of lift changes considerably. Not only does a second frequency appear in the signal, but also the magnitude and phase of $C_{y}$ are more variable. Moving away from the VIV influence towards the upper end of the reduced-velocity range, we note that a higher frequency has appeared in $C_{y}$, although it is not noticeable in the displacement curves.

The upstream cylinder was not only fixed at all times in these experiments, but it was also mounted on a load cell, allowing measurements of instantaneous lift. Analysing the normalized PSD of the lift force on both cylinders, it is possible to identify other branches of frequency in $C_{y}$. Figure 10(a,c) shows the normalized PSD of lift measured on both cylinders. From figure 10(a) it is evident that the lift force acting on the upstream cylinder is directly related to the vortex-shedding mechanism since there is only one distinct frequency branch that follows very closely the $S t=0.2$ line. It can also be concluded that the lift force on the upstream cylinder sees little effect of the oscillation of the downstream one, since only a minor trace of the frequency data presented in figure 8 is identified. On the other hand, figure $10(c)$ shows that the lift force on the downstream cylinder has two clear branches bifurcating after the VIV resonance region. The lower branch corresponds to the frequency of oscillation captured in figure 8, but the higher branch is clearly associated with a vortex-shedding frequency that follows the $S t=0.2$ line.

Now, this vortex-shedding branch is predominant at lower reduced velocities, probably related to the typical synchronization range of VIV, but diminishes beyond $U / D f_{0}=15$. The lower branch appears around a reduced velocity of 5 but only becomes dominant beyond a reduced velocity of 10 . Within the range $U / D f_{0}=10$ 20 , both branches appear with equivalent energy content determining the region where both VIV and WIV are occurring together. In fact, looking again at the response 

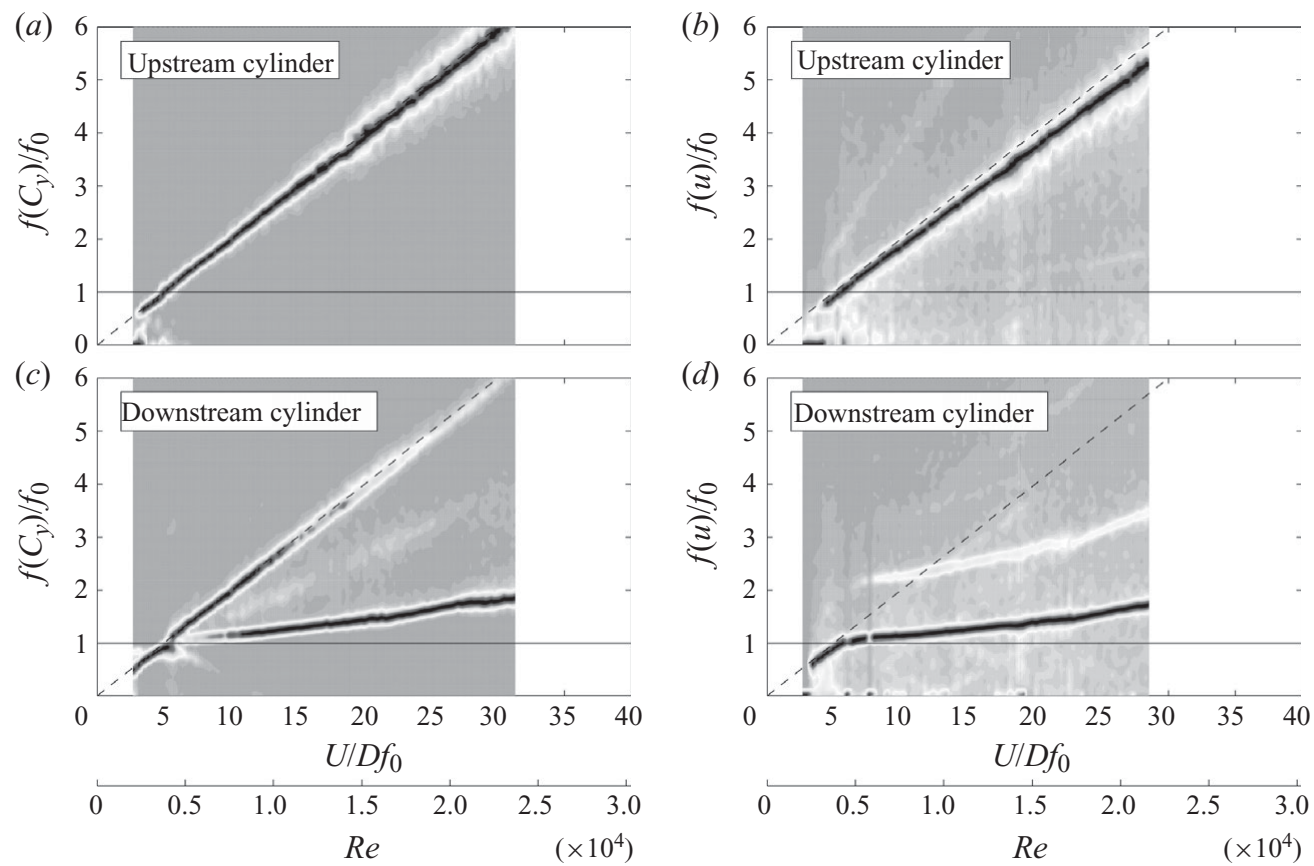

FIGURE 10. Normalized PSD of $(a, c)$ lift force and $(b, d)$ velocity fluctuation in the wake of a static upstream and an oscillating downstream cylinder. (The normalization applied to all PSD graphs does not allow comparison of energy magnitudes along the reduced-velocity axis, but only over vertical slices for a fixed reduced velocity.)

curve in figure 8 , it is quite apparent that three different regimes can be identified by different slopes of the displacement curve: (i) a VIV resonance region around $U / D f_{0}=5$ (equivalent to the VIV upper branch); (ii) combined VIV (reminiscent of a lower branch) and WIV regimes roughly in the range $U / D f_{0}=5-17$; and (iii) a pure WIV regime for $U / D f_{0}>17$.

It is plausible to think that the VIV regime should involve synchronization of vortex shedding from both cylinders. In order to investigate this, we measured velocity fluctuations with hot-film probes, one downstream of each cylinder (see figure 1). Figure $10(b, d)$ presents the PSDs for both probes confirming that the upstream cylinder is shedding vortices as a fixed, isolated cylinder with minimal interference from the downstream one. On the other hand, no clear identification of vortex shedding close to $S t=0.2$ was observed for the downstream cylinder that is oscillating. Of course, once the cylinder is vigorously moving ahead of a fixed probe, it is very difficult to measure any fluctuations other than the component of flow velocity that relates to the fluctuations associated with its movement. However, even when this low-frequency branch was filtered out, no clear trace of vortex shedding was identified. This does not mean that the downstream cylinder is not shedding vortices on the contrary, fully developed vortices were observed in PIV measurements, as will be demonstrated later - it simply means that a hot-film probe positioned in the near wake was not suitable to capture this phenomenon. Placing the probe further downstream made it possible to observe combined velocity fluctuations from the vortex shedding of both cylinders, but still it was not possible to distinguish a shedding signal that could be directly associated with the downstream body. 

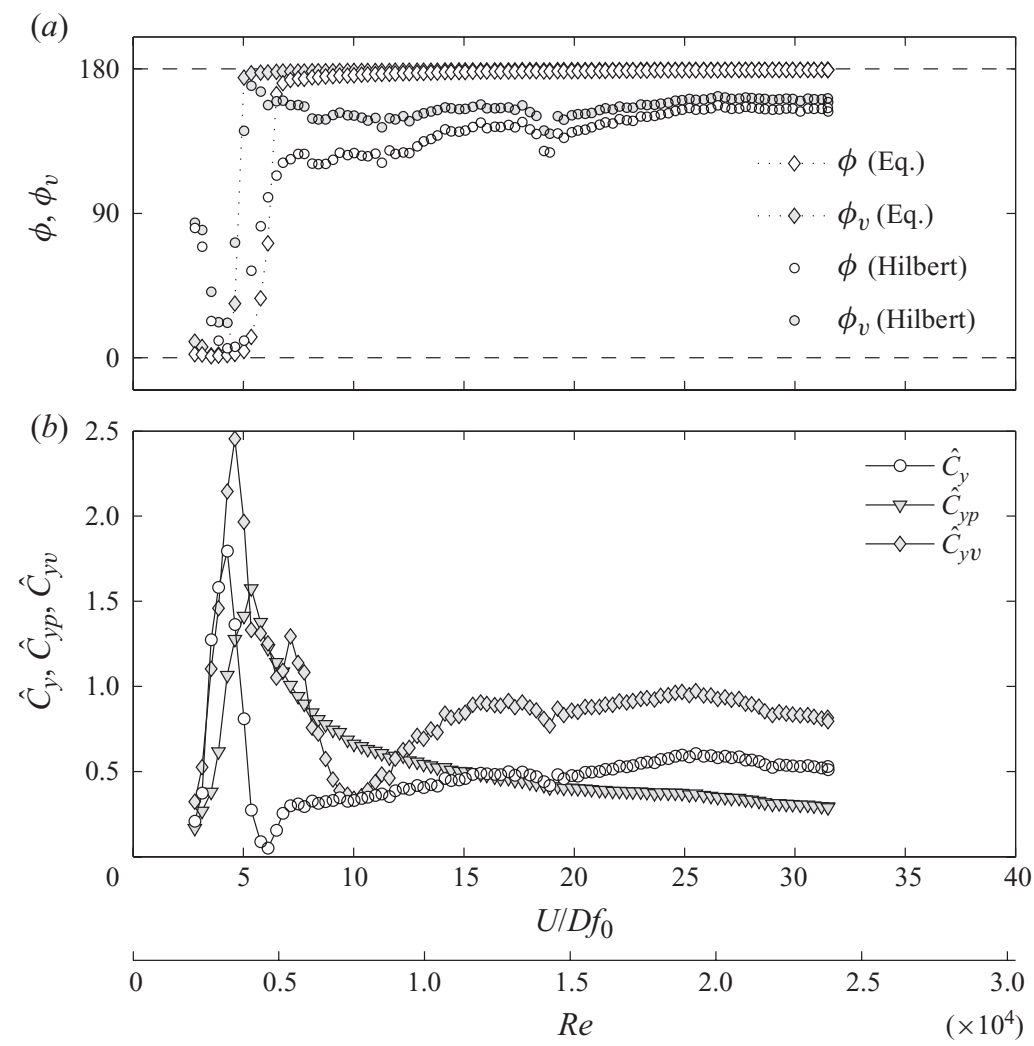

FIGURE 11. (a) WIV phase angles calculated according to Williamson \& Govardhan (2004) and the Hilbert transform of the signals. $(b)$ Decomposition of lift coefficient into potential and vortex components calculated according to Williamson \& Govardhan (2004).

In figure 11 we show the fluid force components acting on the downstream cylinder. In order to estimate $\phi, \phi_{V}, \hat{C}_{y_{V}}$ and $\hat{C}_{y_{P}}$, we have employed a 'harmonic forcing and harmonic motion' hypothesis identical to that presented earlier for VIV of an isolated cylinder, which means that the fluid force and the response have the same frequency but delayed by a phase angle $\phi$. In the pure VIV case, we know that fluctuations in the fluid force come directly from the vortex-shedding mechanism; therefore, $f=f_{s}$ in the synchronization range. However, in the WIV case we have seen that $f$ is not directly related to $f_{s}$ as it does not follow the $S t=0.2$ line closely. Nevertheless, even without knowing the origin of the fluid force, we can verify from figures 8 and 10 that the dominant component of $C_{y}$ has the same frequency as the response. Hence, the harmonic assumption might still throw some light on the phenomenon.

Figure 11 $(a)$ displays $\phi$ and $\phi_{V}$ versus reduced velocity showing that a phase shift from almost $0^{\circ}$ to $180^{\circ}$ occurs at around the same reduced velocity as for typical VIV; beyond the resonance peak, both $\phi$ and $\phi_{V}$ remain close to $180^{\circ}$ until the upper end of the reduced-velocity range. This plot also compares $\phi$ and $\phi_{V}$ calculated by two different methods: the first solves $\phi$ and $\phi_{V}$ from the equations derived in the harmonic analysis of Williamson \& Govardhan (2004) (which also assumes lift and response with a single dominant frequency), and the second averages the phase angles from the instantaneous Hilbert transform for the whole series. If the force and the response indeed present a single harmonic frequency - as they do for singlecylinder VIV - both approaches are equivalent and the curves collapse. However, 

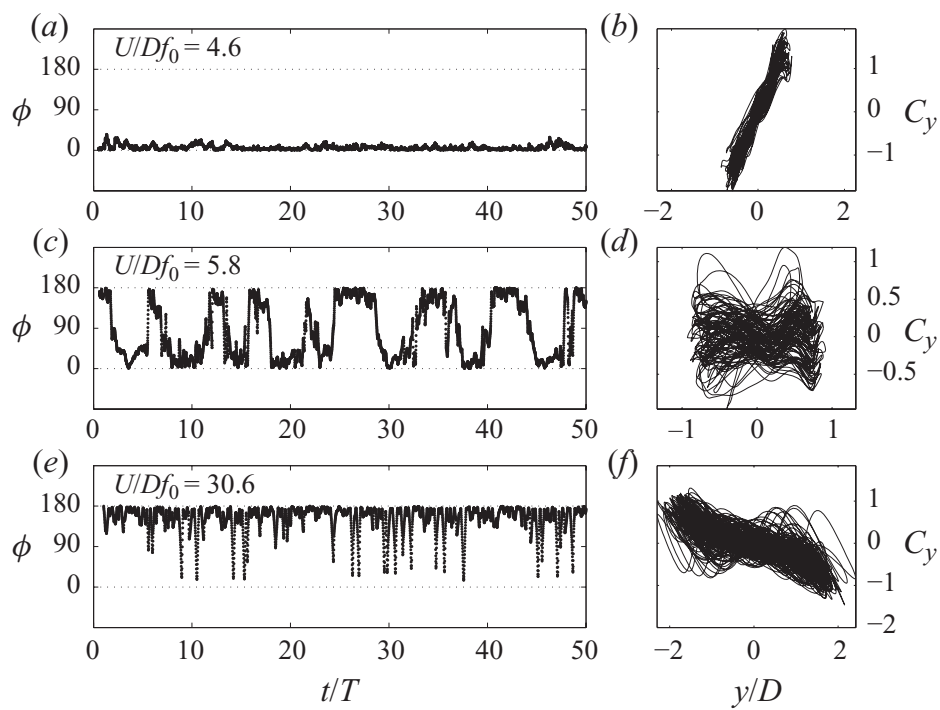

FIGURE 12. Three examples of WIV phase angles. $(a, c, e)$ Instantaneous phase angle $\phi$ for around 50 cycles of oscillation. $(b, d, f)$ Lissajous figures of $C_{y}$ versus $\hat{y} / D$.

figure 11(a) shows that the harmonic hypothesis must be an over-simplification of the WIV phenomenon. The actual phase angles calculated with the Hilbert transform of the displacement and lift signals are at a lower level for the whole range of the WIV excitation. This result reveals that the lag between displacement and lift is actually less than the one predicted by a harmonic assumption.

Figure 11(b) shows the decomposition of the lift coefficient. For reduced velocities up to the VIV resonance peak, the curves show behaviour very similar to that found for single-cylinder VIV. But instead of $C_{y}$ and $C_{y_{V}}$ reducing and tending to zero by the end of the synchronization range, both rise from around a reduced velocity of 7 up to 17, marking the second regime of combined VIV (a possible lower branch) and WIV. A clear WIV regime is identified in $C_{y}$ and $C_{y_{V}}$ curves for $U / D f_{0}>17$, as mentioned above, and their values remain roughly at the same level as reduced velocity is increased.

The instantaneous behaviour of $\phi$ or $\phi_{V}$ may be analysed by employing a Hilbert analytical transform to the signal, as described in Hahn (1996) and employed by Khalak \& Williamson (1999) for VIV. Figure 12 presents the results for about 50 cycles of oscillation. $\phi$ remains very close to $0^{\circ}$ for the whole time series at $U / D f_{0}=4.6$, resulting in a clean Lissajous figure in figure $12(b, d, f)$. During the transition at $U / D f_{0}=5.8$, it appears that an intermittent phase shift is also present, consistent with the data plotted in figure 9. When the regime reaches our highest reduced velocity of $U / D f_{0}=31.2$, the phase is predominantly close to $\phi=180^{\circ}$, but it still varies more than for VIV of a single cylinder. The corresponding Lissajous figure may suggest that a second dominant frequency may also be playing a role, in agreement with the third case analysed in figure 9.

\section{The wake-induced vibration excitation mechanism}

In order to understand the fluid mechanics behind the WIV mechanism, we investigated two aspects of the force acting on the downstream cylinder: firstly, the origin of the restoring lift force occurring on static cylinders in staggered 
(a)

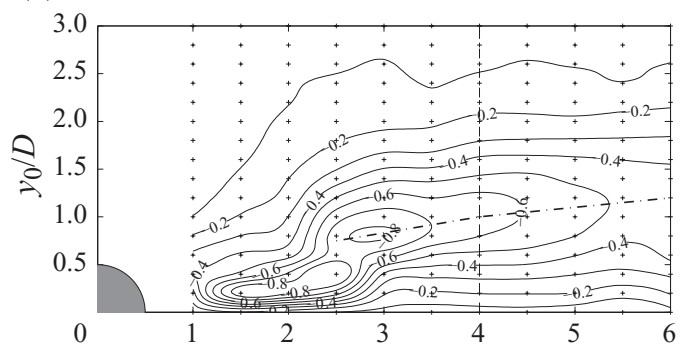

(c)

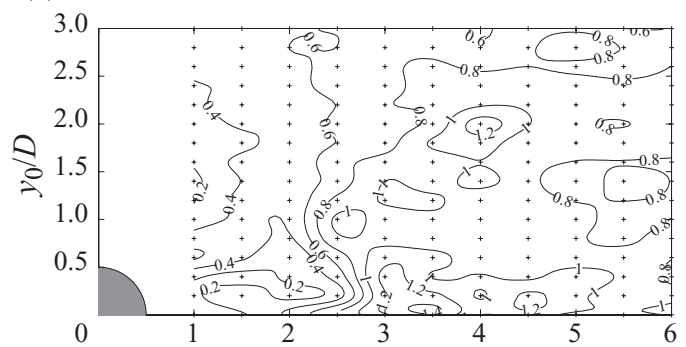

(e)

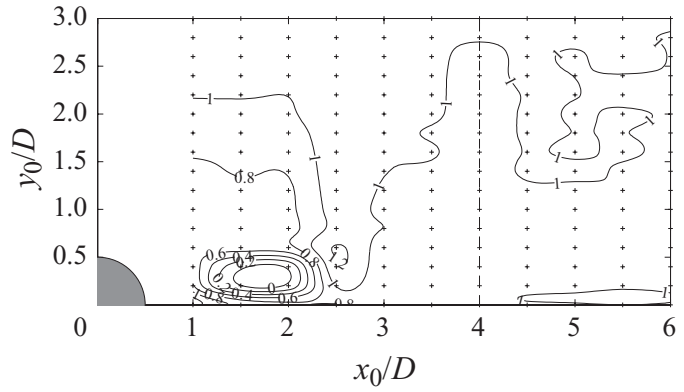

(b)

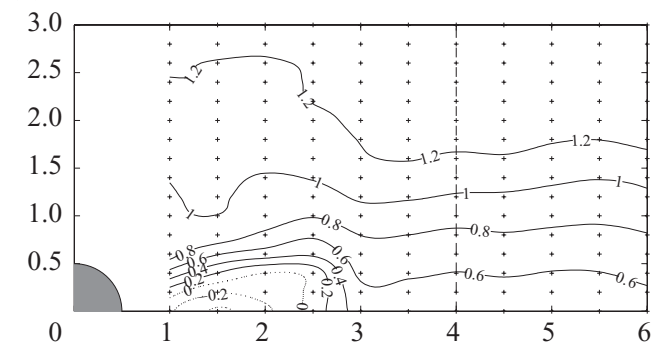

(d)

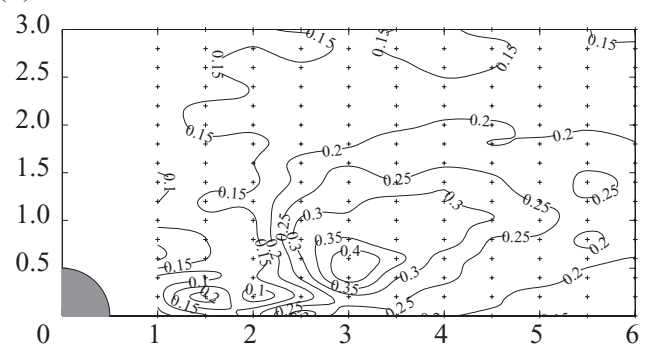

$(f)$

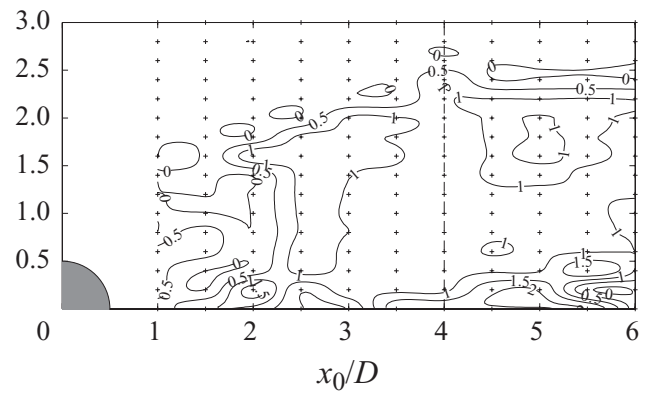

FIGURE 13. Contours for the downstream cylinder of a static pair at $R e=19200$ : $(a)$ steady lift $\left(\bar{C}_{y}\right) ;(b)$ steady drag $\left(\bar{C}_{x}\right) ;(c)$ fluctuating lift $\left(\hat{C}_{y}\right) ;(d)$ fluctuating drag $\left(\hat{C}_{x}\right) ;(e)$ frequency of lift $\left(f\left(C_{y}\right) / f_{s}\right) ;(f)$ frequency of drag $\left(f\left(C_{x}\right) / f_{s}\right)$.

arrangements; secondly, the origin of the phase lag between $C_{y}$ and $y$ that, combined with the lift field, results in the WIV excitation.

\subsection{Fluid force on static cylinders in staggered arrangement}

Measurements were obtained by holding the upstream cylinder fixed and traversing the downstream cylinder across 160 stations (each marked by a small cross in figure 13) in and out of the wake-interference region. Results are presented in figure 13 in a series of maps that are symmetrical about the centreline of the wake.

Figure 13(a) presents the steady lift acting on the downstream cylinder for different regions of wake interference. A negative value of $\bar{C}_{y}$ indicates lift acting towards the centreline. As expected, the first evident observation is that the steady-lift force points in the direction of the centreline for all configurations investigated, which is in agreement with Bokaian \& Geoola (1984) and Zdravkovich (1977). The $\bar{C}_{y}$ map reveals two regions of steady lift as large as -0.8 . The first region, between $x_{0} / D=1.5$ and 2.5 , is associated with the gap-flow-switching mechanism described by Zdravkovich (1977) and occurs in the first wake-interference regime. The second 
region occurs for larger lateral separations around $y_{0} / D=0.8$; it begins around $x_{0} / D=2.5-3.0$ and develops a trend of maximum $\bar{C}_{y}$ (indicated by the dot-dashed line) that will decrease in intensity as the second cylinder is moved farther downstream. This second region is associated with the second interference regime, in which vortex shedding takes place in the gap.

In the steady drag map presented in figure $13(b)$, positive contours of $\bar{C}_{x}$ denote drag in the streamwise direction. Dotted lines represent contours of zero or negative drag that occur when the cylinders are close enough for the gap flow to be enclosed by the reattaching shear layers. For $x_{0} / D>2.5$, the tandem downstream cylinder only experiences positive drag, indicating that a developed wake starts to be formed in the gap.

If a quasi-steady assumption is to be used to understand WIV, then these maps of time-average force coefficients should be adequate to provide the necessary gradients of $C_{y}$ and $C_{x}$ to satisfy a classical-galloping-like model. However, by also analysing the fluctuating components of the fluid force, it is possible to identify if and where the unsteadiness of the flow is playing a significant role.

Figures $13(c)$ and $13(d)$ present maps similar to those discussed above, but plot contours of the fluctuating-force coefficients $\hat{C}_{y}$ and $\hat{C}_{x}$ (3.3). Both graphs reveal regions of increased fluctuating lift and drag that only occur for $x_{0} / D>2.5$. A contour of $\hat{C}_{y}>1.0$ appears for tandem arrangements but relatively high values of $\hat{C}_{y}>0.8$ also appear for staggered locations around the wake-interference region. $\hat{C}_{y}$ is reduced to levels below 0.4 for separations below the critical value. Interestingly, the region of maximum $\hat{C}_{x}$ does not occur for tandem arrangements but only when the downstream cylinder has an offset of about $y_{0} / D=0.5$. $\hat{C}_{x}=0.4$ is observed for the second interference regime at $x_{0} / D=3.0$ and a trend of higher fluctuating drag is developed from this point, decreasing in intensity as $x_{0}$ is increased.

The distribution of $\hat{C}_{y}$ and $\hat{C}_{x}$ across the wake gives support to the idea that coherent vortices from the upstream cylinder contribute to the fluctuating component of the fluid forces for separations of $x_{0} / D>3.0$. The magnitude of $\hat{C}_{y}$ is another important factor. Taking the example of $x_{0} / D=4.0$ and $y_{0} / D=1.0$, we observe that the magnitude of the fluctuating lift is greater than that of the steady lift, i.e. the actual lift on the cylinder is $C_{y}=-0.6 \pm 0.8$, probably even reaching an instantaneous positive (outwards) value once in a few cycles.

We believe this to be strong evidence that the steady-lift force acting towards the centreline, as well as the fluctuating component, originate in some way from the unsteady interference of the vortex wake coming from the upstream cylinder with the wake being formed from the downstream cylinder. If this is true, we expect to find that the frequency of fluctuation of $C_{y}$ and $C_{x}$ is related to the frequency of vortex shedding from upstream. Now, we know that the upstream cylinder is shedding vortices at the same frequency as an isolated cylinder (see figure 10). Figures 13(e) and $13(f)$ show plots of the frequencies of $C_{y}$ and $C_{x}$, respectively (normalized by the equivalent $f_{s}$ for $S t=0.2$ ). We observe that the dominant component of the fluctuating forces is close to 1 once the second regime of interference is established. For close separations, the frequency of $C_{y}$ is distinctively lower than $f_{s}$, revealing that a developed wake is indeed not present in the gap. This result is in agreement with Alam et al. (2003), who measured lift coefficients for both cylinders in tandem arrangements.

Our experimental results for the steady components are in good agreement with other works found in the literature, including Zdravkovich (1977). We believe this is the first time the magnitude and frequency of the fluctuating components of the lift and drag forces have been presented for staggered arrangements of cylinders. 


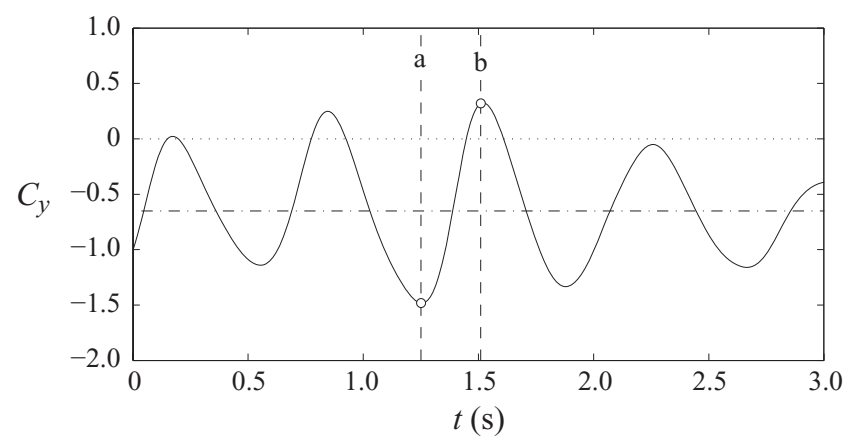

FIgURE 14. Time series of lift on the downstream cylinder of a static pair in staggered arrangement $x_{0} / D=4.0$ and $y_{0} / D=1.0 . R e=19200$.

Now, a quasi-static analysis of WIV requires the downstream cylinder to extract energy from the flow as it moves across the steady-force fields discussed above. In other words, the excitation force acting on the body should not depend on its movement or any unsteady interaction with the upstream wake, but only on the relative position of the cylinder across the wake. This might be true for bodies with very small transverse velocities $\dot{y}$, but certainly this is not the case observed in the WIV response presented above. Even a quasi-steady hypothesis may be too much of an over-simplification in this case. Based on data from figure 8 , we can estimate the maximum transverse speed the cylinder reaches as it crosses the centreline of the wake for the maximum reduced velocity point. On average, $\dot{y}$ is around $55 \%$ of the free-stream velocity $U$, but it can reach values up to $67 \%$ for the most severe cycles. With such vigorous crossflow movement, it is difficult to accept that the downstream cylinder is not affecting or interacting with the wake coming from the upstream body, making implausible any quasi-static or quasi-steady assumptions. Therefore, we believe that a completely unsteady investigation of the force-displacement interaction is required to understand how the WIV mechanism works.

\subsubsection{Analysis of unsteady lift on fixed cylinders}

Before investigating the instantaneous lift force acting on a moving downstream cylinder, we shall consider the unsteady flow field that generates the steady and fluctuating forces on a pair of static cylinders.

Figure 14 shows a short time series of $C_{y}$ measured on a static downstream cylinder at $x_{0} / D=4.0$ and $y_{0} / D=1.0$. The dot-dashed line represents a steady lift of $\bar{C}_{y}=-0.65$ estimated from figure 13 . There are two data points, marked with circles, representing the maximum and minimum $C_{y}$ in this short time series for which we will investigate the corresponding flow fields using PIV. Figure 15 shows instantaneous vorticity contours and the corresponding velocity field for instant ' $a$ ' in the trough, i.e. when $C_{y}=-1.4$, and for instant 'b' at the crest, where $C_{y}=0.32$ and acts outwards. Vortices identified with the symbols A and B were, respectively, shed from the upstream and downstream cylinders; odd indices mean that vortices have positive vorticity and were shed from the right-hand sides of the bodies, and even indices mean the opposite. Vortices are identified at both instants; therefore, we can follow the development of the wake from ' $a$ ' to ' $b$ '. A simplified sketch is presented in figure 16.

The flow passing around the upstream cylinder generates and sheds vortices in the gap. In figure 15, we see the instant when vortex A4 is being formed very 


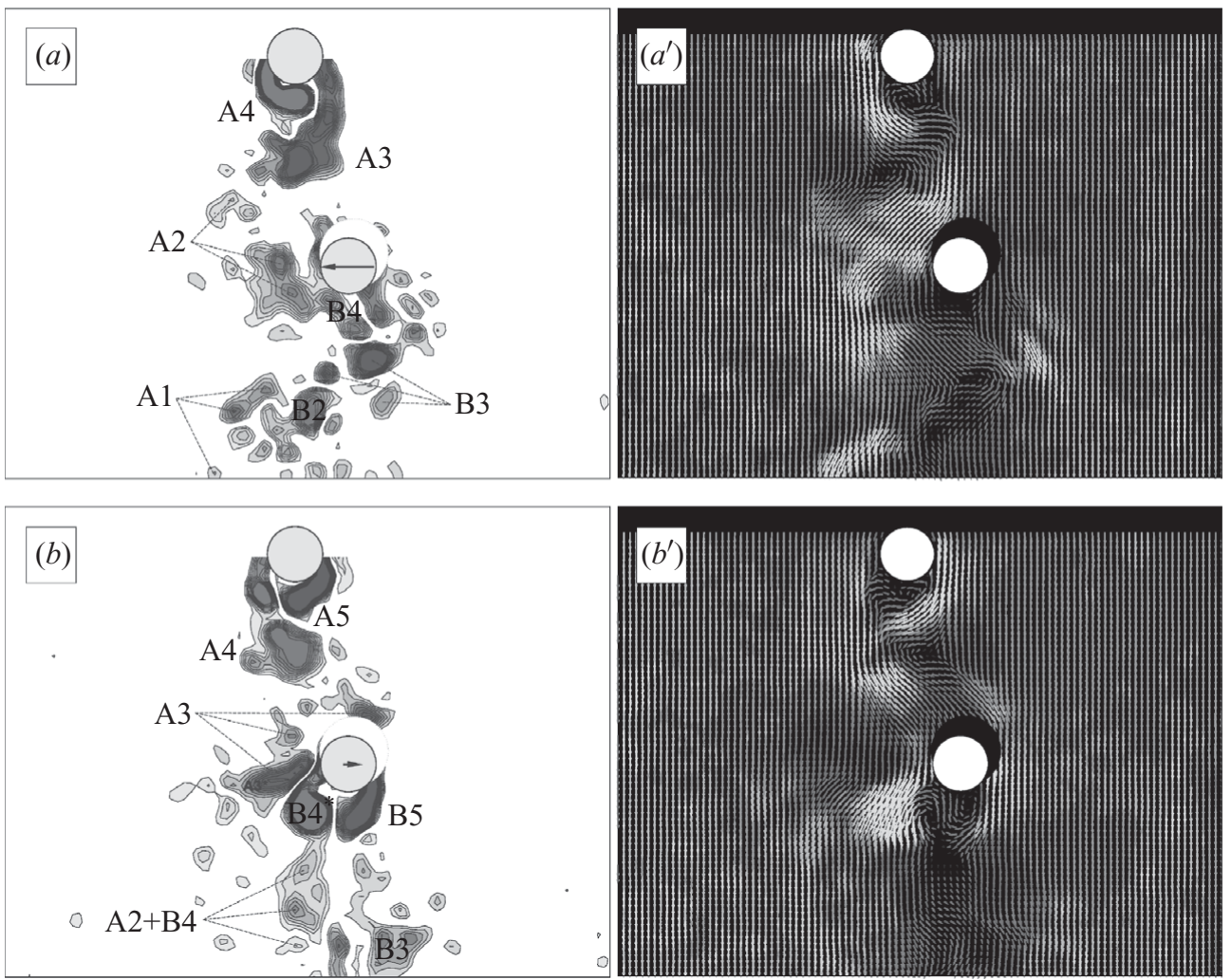

FigURE 15. $(a, b)$ Instantaneous vorticity contours and $\left(a^{\prime}, b^{\prime}\right)$ velocity field (velocity magnitude increases from dark to light grey) obtained with PIV around a pair of static cylinder in staggered arrangement. $x_{0} / D=4.0, y_{0} / D=1.0, R e=19200$. (See figure 14.)

(a)

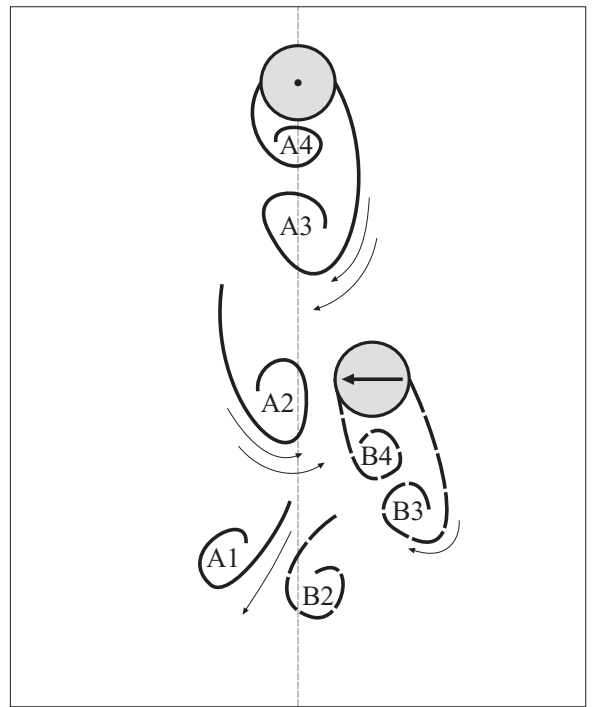

(b)

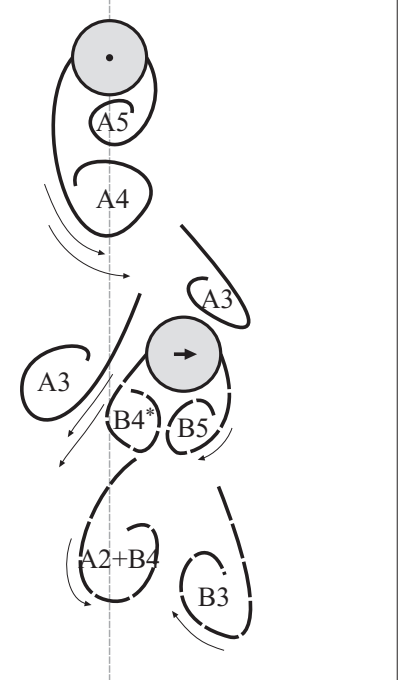

FIGURE 16. Sketch of vortex-structure interaction that $(a)$ enhances or $(b)$ diminishes $C_{y}$ on the downstream cylinder at $x_{0} / D=4.0$ and $y_{0} / D=1.0$. (See figures 14 and 15.) 
close to the cylinder, inducing a high-speed flow that is shown with white vectors in the velocity field. A fully developed vortex A3, which was formed half a cycle before, is convected downstream and induces high-speed flow on the inner side of the downstream cylinder. This high-speed flow accelerates the boundary-layer flow running on that side, adding more circulation into the shear layer. Consequently, vortex B4 forming from the downstream cylinder will have a higher circulation than a typical vortex on a single-cylinder fully developed vortex A2, shed in the previous cycle, passes around the downstream cylinder and induces a flow field that will hold B4 closer to the downstream cylinder. We suggest that this combination of high-speed flow induced on the inner side, increasing the strength of the vortex being formed, and the flow holding this vortex closer to the downstream cylinder is responsible for generating the high lift of $C_{y}=-1.4$ shown in figure 14. Of course, the interaction between vortices from previous cycles is occurring in the wake downstream of the second body - with vortices from both cylinders (A1 and B2) merging together and moving in pairs - but what is happening around the downstream cylinder has significantly more influence on the force being generated than the wake further downstream.

Now, moving to instant ' $b$ ' in figure 15 , we observe that vortices have been convected further downstream and a new vortex A5 is being formed from the upstream cylinder. Downstream of the second cylinder, we observe that A2 has merged with B4, forcing B3 to be released and giving way to a new B4* that starts to roll up. A fully developed A3 impinges and splits around the downstream cylinder with a portion A3* passing by the inner side and the rest following around the outside of the cylinder. As A3* and the new B4* interact, they induce a very high-speed flow across the wake that forces the formation of $\mathrm{B} 4 *$ further inwards. On the other side, B5 is also forced and rolls up closer to the downstream cylinder, contributing to generating a small lift $C_{y}=0.32$ acting outwards.

Figure 16 highlights the main vortex-structure interactions occurring in the wake. The effect of upstream vortices on the downstream cylinder is seen to be paramount in both cases: $(a)$ when $\mathrm{A} 3$ induces high-speed flow on the inner side and A2 displaces the downstream wake outwards and $(b)$ when $\mathrm{A} 3$ splits around the body and interacts with B4 to generate high-speed flow inwards.

We observe that identical wake patterns are not repeatable, as vortices from both cylinders may be forming at different rates and strengths. Nevertheless, we believe this flow-field investigation offers a good illustration of the complex vortex dynamics occurring in the wake associated with large lift fluctuations on the downstream cylinder. We should expect to find even more complex dynamics when the downstream cylinder is oscillating with high transverse velocities.

We saw that a strong and complex vortex-structure interaction is present, and hence, must be involved in the excitation mechanism. We cannot guarantee a priori that a quasi-steady assumption for the fluid forces (even if it accounts for hysteretic effects) will represent the phenomenon with all its unsteadiness. Instead, we suggest that a phase lag is generated as the unsteady wake is modified by the movement of the downstream cylinder. Hence, we propose that a simple steady wake without the unsteadiness of the vortices is not able to generate the necessary forces and phase lag to excite WIV. In order to evaluate this hypothesis, we will proceed in two steps. Firstly, we present an idealized experiment designed to reproduce a wake with a steady shear-flow profile but without the unsteadiness of vortices. Then we will investigate lift-force measurements paired with instantaneous flow fields to assess whether there 


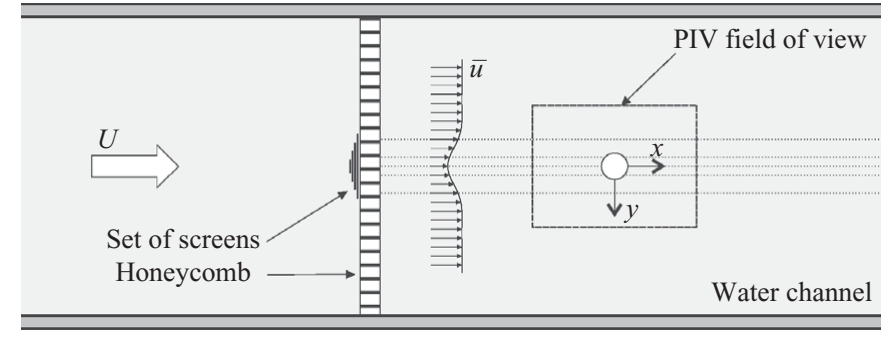

FIGURE 17. Set-up of set of screen and honeycomb for the shear-flow experiment.

is a phase lag related to the vortex-structure interaction as the downstream cylinder oscillates.

\section{The effect of a steady shear flow}

If a quasi-steady approach is to be used to predict WIV then the fluid forces on the downstream cylinder will not depend on the unsteadiness of the wake but only on the steady flow velocity profile. Therefore, if we could generate a wake with a similar steady profile but without the unsteadiness of the vortices and immerse an elastically mounted cylinder in this velocity field, we should expect to see a response similar (at least qualitatively) to WIV. According to the quasi-steady approach, the phase lag between lift and displacement would still have to be present in such a flow. A qualitatively similar WIV response would be sound evidence that unsteady vortices are not required to generate the phase lag and sustain vibrations.

\subsection{Experimental set-up}

A series of screens made of thin stainless-steel wire was cut in strips of different widths. Then a combination of superimposed screens was positioned vertically in the centre of the test section to produce a mean velocity profile. The set of screens was fixed on an aluminium honeycomb to remove any crossflow components. The set-up is illustrated in figure 17 , where $\bar{u}$ represents the resultant shear-flow profile. The flow field around a pair of cylinders under WIV was measured with PIV to serve as reference. The velocity profile of the wake was averaged from a number of snapshots (corresponding to more than 100 cycles of oscillation and many more cycles of vortex shedding), resulting in the flow fields presented in figures 18(a) and 18(b). The process was repeated for four $R e$ within the range of the experiments, although only two are plotted here for brevity. Two dashed circles mark the average amplitude of oscillation of the cylinder.

When considering fluid forces acting on the downstream cylinder Price (1976) had already noticed that the lift profile on the body depends 'on its own characteristics in the wake and not particularly on the wake characteristics'. He concluded that 'the use of wake parameters, measured without the presence of the [downstream] body when attempting to assess the forces thereon, is an over-simplification of the situation as far as lift is concerned, while appearing to work quite well for drag'. For this reason, flow-field measurements were performed with the downstream cylinder in place and oscillating in order to account for the interaction between the body and the upstream wake.

With the cylinders removed, the set of screens was adjusted to generate the best possible match to the profiles of the reference cases. Figures $18(c)$ and $18(d)$ present the steady profiles obtained downstream of the screens for two of the Re investigated. 
(a)

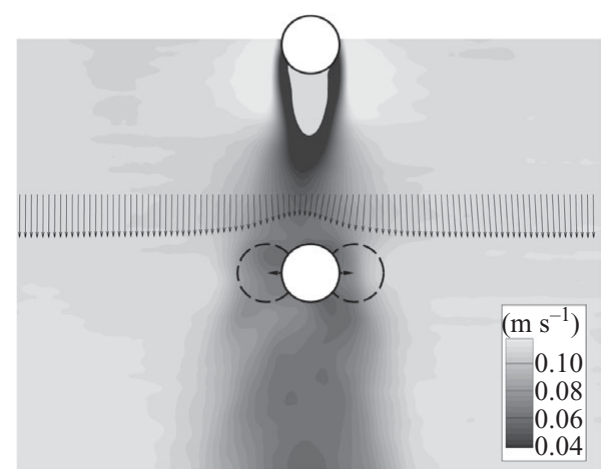

(c)

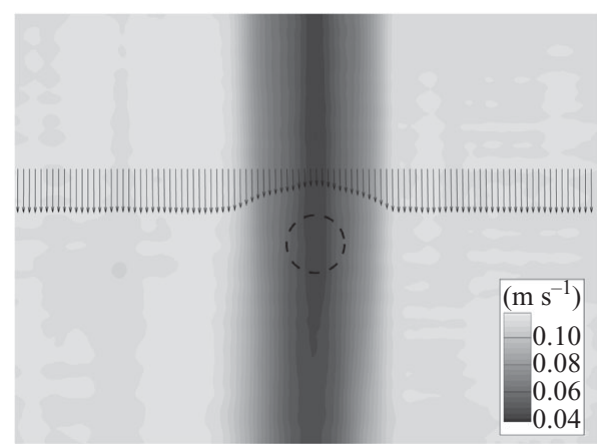

(b)

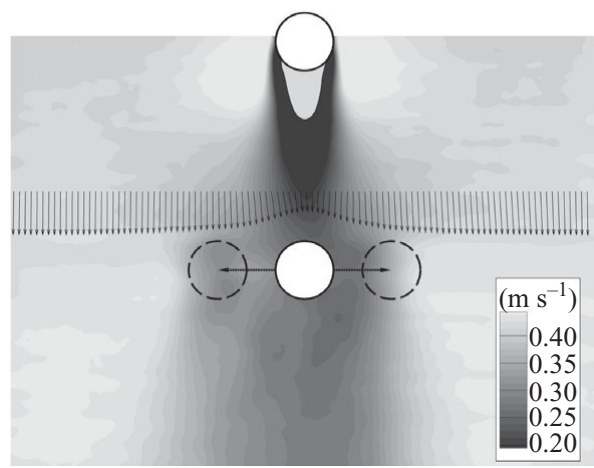

(d)

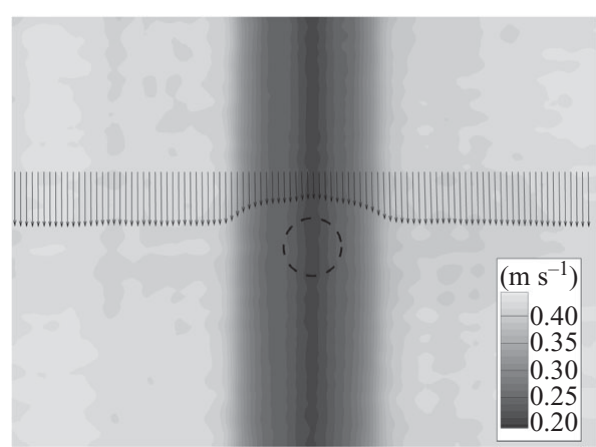

FIGURE 18. Steady flow velocity field $(a, b)$ around a pair of cylinders while the downstream cylinder is oscillating under WIV, and $(c, d)$ generated by a set of screens (Re based on the cylinder diameter). Contours of $\bar{u}$ coloured by velocity magnitude. $(a, c) R e=4800 ;(b, d)$ $R e=19200$.

The dashed circle represents the position where the cylinder would be placed. The streamwise velocity profile across the wake at $x_{0} / D=3.0$, represented by an array of vectors plotted across the wake in the gap, was extracted from the PIV flow fields and used to validate the comparison. The result is plotted in figure 19 for the four $R e$ investigated. The average breadth of the wake and the minimum flow speed on the centreline were the main wake parameters employed to calibrate the screens. Nevertheless, the geometry was optimized to guarantee that the correlation coefficients between corresponding profiles were above $R=95 \%$,

$$
R=\frac{1}{n} \sum_{i=1}^{n}\left(\frac{u_{1_{i}}-\overline{u_{1}}}{\sigma_{u_{1}}}\right)\left(\frac{u_{2_{i}}-\overline{u_{2}}}{\sigma_{u_{2}}}\right)
$$

where $\bar{u}$ and $\sigma_{u}$ represent the mean and standard deviation, respectively, of the $n$ points defining each velocity profile $u_{1}$ and $u_{2}$. Figure 19 reveals a reasonably good agreement between reference and generated profiles in spite of the complexity of the set-up designed to achieve these results.

An instantaneous snapshot of the vorticity field plotted in figure 20 reveals that the shear profile generated by the screens does not have the coherent vortices typical of bluff-body vortex shedding. This is even clearer when the instantaneous wake is 


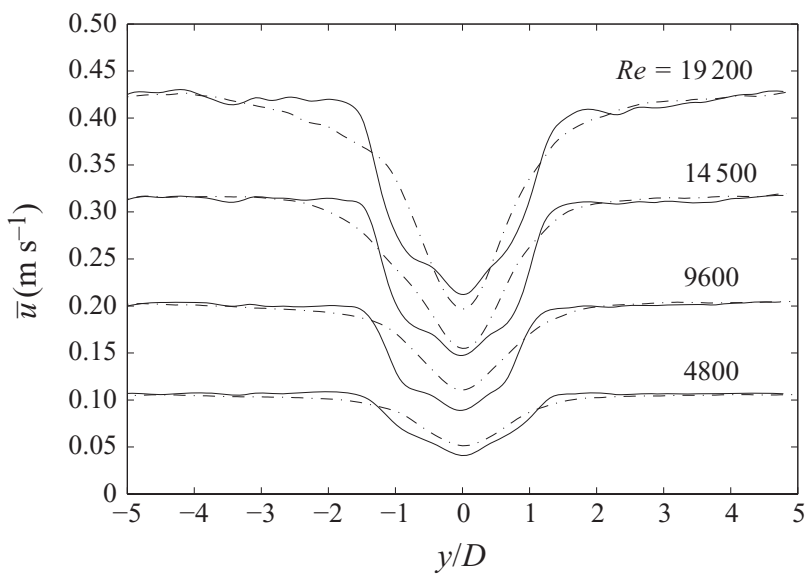

FIGURE 19. Comparison between steady velocity profiles for various flow speeds measured across the wake at $x_{0} / D=3.0:-$, set of screens; $-\cdot-$, pair of cylinders. $R=98,97,97$ and $96 \%$ from the lowest to the highest $R e$.

(a)

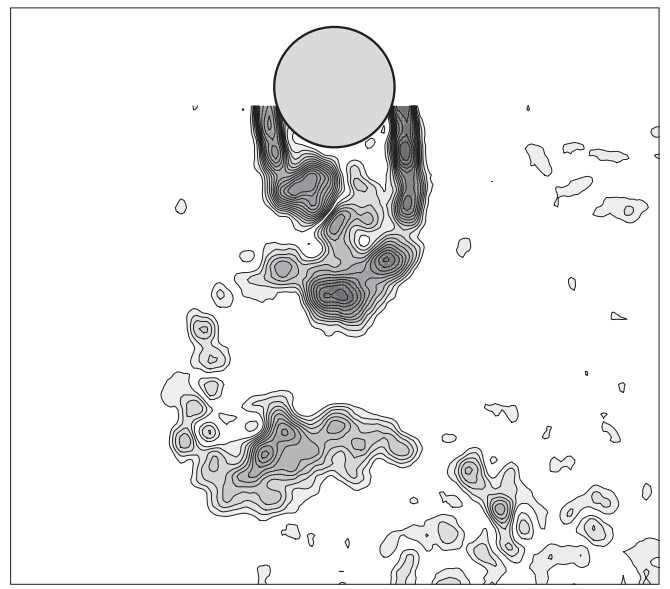

(b)

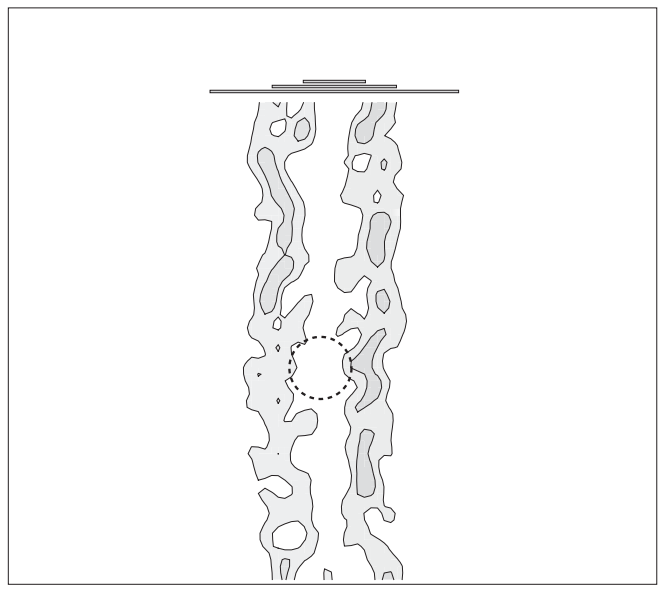

FIGURE 20. Instantaneous vorticity contours of the wake downstream of $(a)$ a single cylinder and $(b)$ a set of screens. Both images have the same contour-colour scale to allow direct comparison. $R e=9600$ based on cylinder diameter.

contrasted with the wake of a static-cylinder shedding vortices in the $2 \mathrm{~S}$ mode. Both vorticity fields in figure 20 have the same contour-colour scale, with dark-grey colour meaning greater vorticity, allowing direct comparison between fully developed wakes. (The position of the screens in figures 18 and 20 is merely illustrative. In reality, the set of screen was positioned at around $10 \mathrm{D}$ upstream of the cylinder, as shown in figure 17.)

\subsection{Steady forces on a static cylinder}

We begin by investigating the steady fluid forces acting on a static cylinder as it traverses across the shear flow for various Reynolds numbers. Figure 21( $a, c)$ shows maps of lift and drag that can be compared to the steady-force maps of a pair of staggered cylinders (extracted from figure 13). We immediately see a considerable 

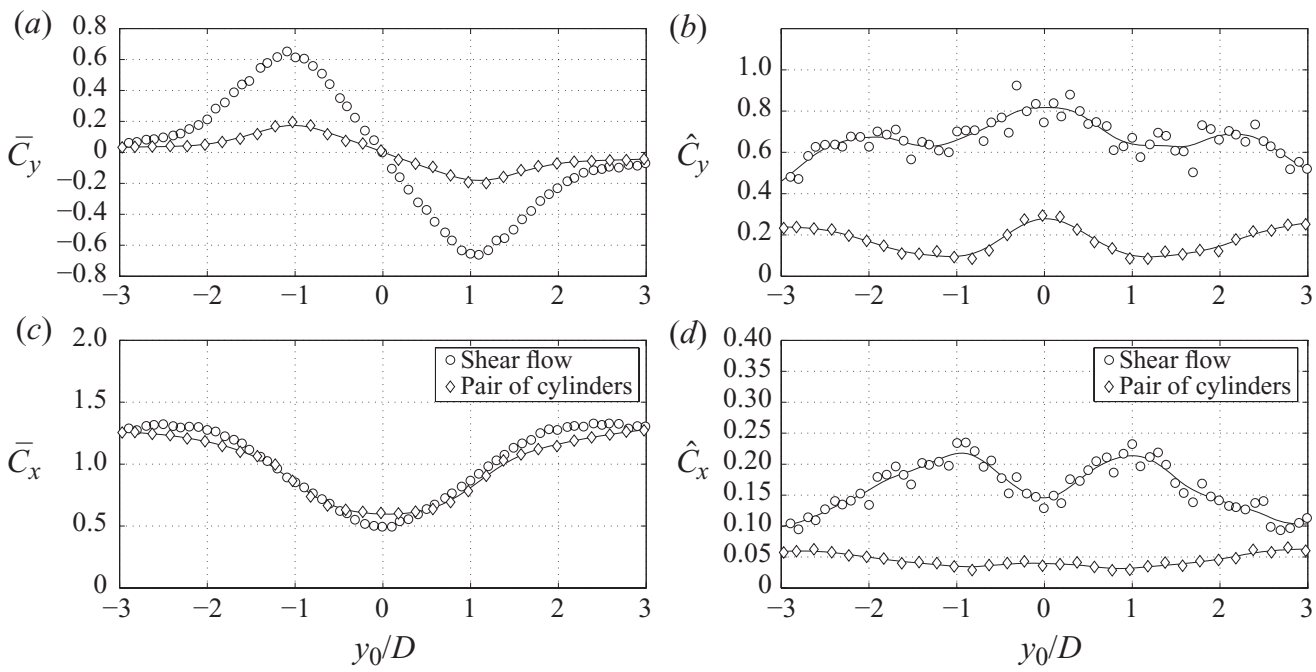

FIgURE 21. Steady $(a, c)$ and fluctuating $(b, d)$ fluid forces on a static cylinder in shear flow and on the downstream cylinder of a pair with $x_{0} / D=4.0$. $R e=19200$.

difference between the lift curves. While for a pair of cylinders the minimum lift towards the centreline reached $\bar{C}_{y}=-0.65$ at around $y_{0} / D=1.0$, the cylinder immersed in a shear flow only reaches a minimum of $\bar{C}_{y}=-0.2$ for the same position. Nevertheless, the similarity in steady drag between the two cases is remarkable. Both reach a minimum around $\bar{C}_{x}=0.5$ on the centreline of the wake with a very similar behaviour as the cylinder is displaced outwards. This correspondence must be related to the fact that the streamwise component in both cases is very similar; therefore, the shielding effect observed in the steady flow field is well reproduced by the screens.

In addition, we also note a remarkable reduction in the fluctuating fluid forces if vortices are not present in the upstream wake. Figure $21(b, d)$ compares $\hat{C}_{y}$ and $\hat{C}_{x}$ on static cylinders for both experiments. While the fluctuating lift coefficient reaches values around $\hat{C}_{y}=0.8$ for the downstream cylinder of a tandem pair, the maximum fluctuation of lift in the shear flow is only around $\hat{C}_{y}=0.3$, which is very close to the magnitude of $\hat{C}_{y}=0.35$ due to vortex shedding measured for a single static cylinder. The fluctuation in drag is also affected, with a 10 -fold amplification at $y_{0} / D=1.0$ if vortices are present in the upstream wake. Note that for larger separations out of the wake-interference region, the curves for both cases seem to be converging to the value of an isolated cylinder.

This is strong evidence suggesting that vortex interactions from the upstream wake are responsible for the high steady and fluctuating lift on static cylinders in staggered arrangements. Remove the unsteadiness from the wake and the steady lift towards the centreline is considerably reduced, almost disappearing, with the fluctuating term tending towards values measured for a single cylinder.

Previous works tried to attribute the existence of a steady force towards the centreline to the other mechanisms, as summarized by Price (1976), but none of those mechanisms accounted for the total magnitude of $\bar{C}_{y}$ on the downstream cylinder. In figure $21(a, c)$, we showed that a residual $\bar{C}_{y}=-0.2$ at $y_{0} / D=1.0$ still remains even when vortices are removed from the upstream wake. Of course, not all the unsteady vorticity in the wake could be removed, as seen in the instantaneous flow contours 


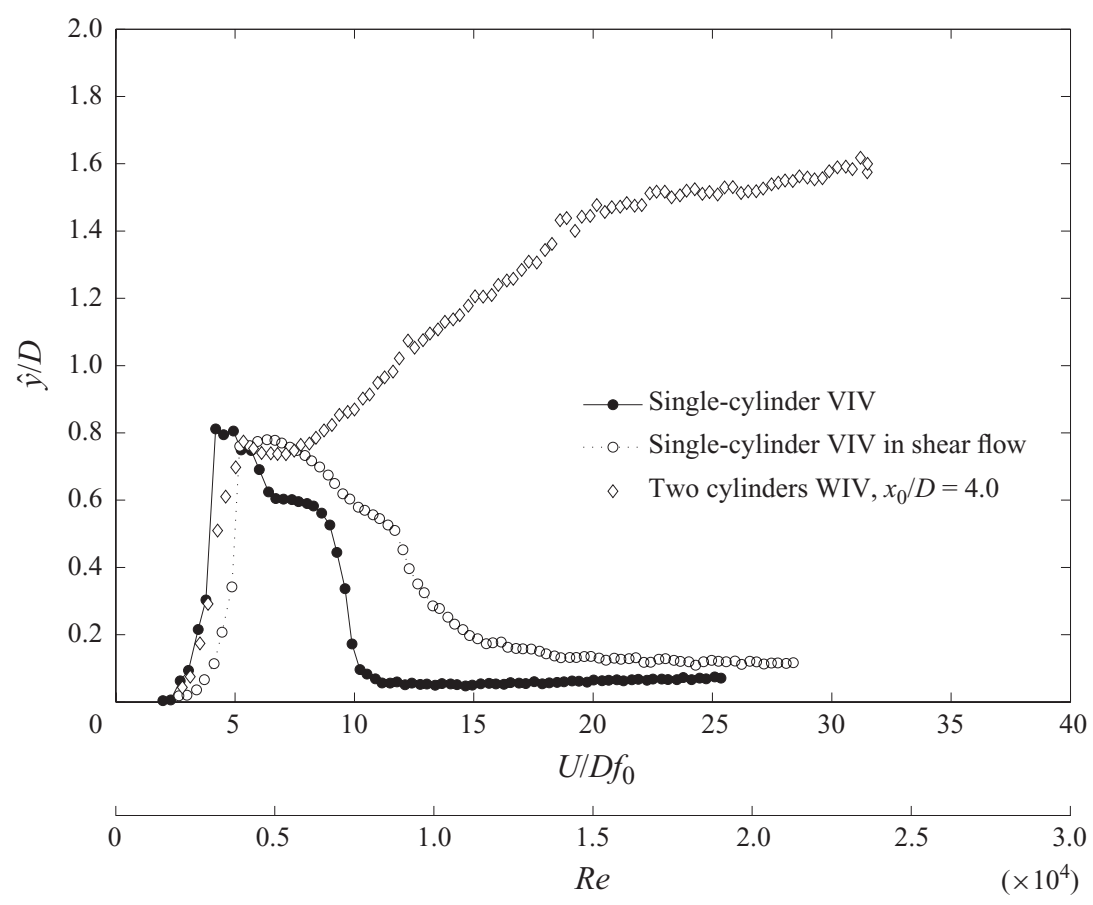

FIGURE 22. Response of the downstream cylinder in shear flow compared with typical VIV and WIV responses.

of figure 20, and the residual $\bar{C}_{y}$ towards the centreline may as well be an effect of this weak unsteady vorticity field that still remains. Nonetheless, we were able to show that the presence of vortices is indeed responsible for the high $\bar{C}_{y}$ measured for staggered cylinders. In summary, the presence of a steady shear flow contributes to a minimum $\bar{C}_{y}=-0.2$, but only with the presence of unsteady vortices will the total $\bar{C}_{y}=-0.65$ be reached - not to mention the effect of unsteady vortices in enhancing the fluctuating term of the fluid force presented in figure $21(b, d)$. This result combined with the unsteady analysis presented in figure 15 offers a good explanation for the role of vortical structures in enhancing lift on the downstream body.

\subsection{Response of an elastic cylinder in shear flow}

We then placed an elastically mounted cylinder in the shear flow in order to investigate the FIV response. However, the response that built up was completely different from previous WIV results. Figure 22 shows the comparison.

Instead of developing a high-amplitude branch that increases with reduced velocity, the response resembled that of a single cylinder under VIV. A clear resonant peak is observed around $U / D f_{0}=7$ (note that here $U$ is the free-stream velocity outside the shear flow), but drops steeply towards a residual level below $\hat{y} / D=0.2$ for reduced velocities higher than 15. Even though upper and lower branches are not as clearly distinguishable as for a single-cylinder VIV, a synchronization range is still evident. The response is slightly different from the typical VIV curve, but it is strikingly similar to VIV rather than to the measured WIV curve also plotted in figure 22. In fact, the similarity is so strong that we cannot avoid concluding that the cylinder in shear flow is only responding with a type of VIV modified by the steady shear flow. The 


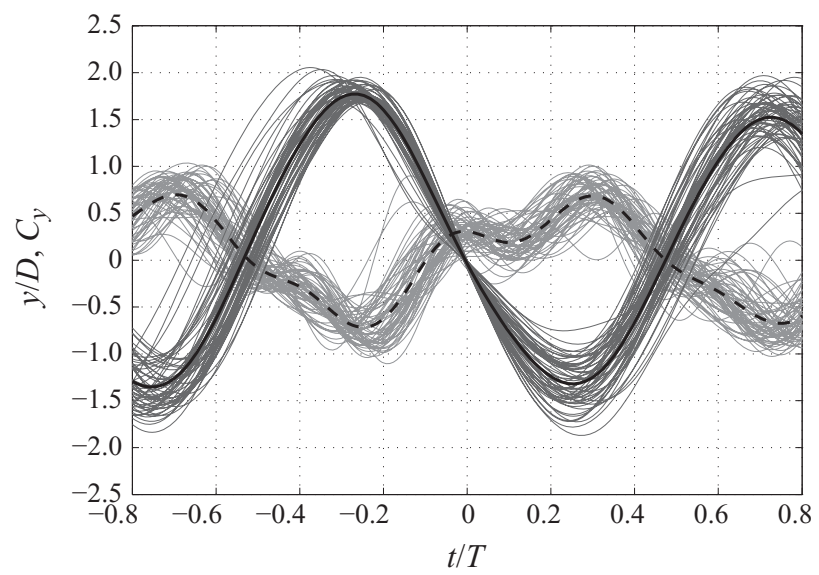

FIGURE 23. Superimposed plots of WIV cycles with similar amplitude; $y / D$ in dark grey and $C_{y}$ in light grey with average cycle in black. $x_{0} / D=4.0, U / D f_{0}=25$ and $R e=19200$.

fact that the resonance peak is slightly offset towards higher reduced velocities is in agreement with the shielding effect of the steady wake. From the velocity profiles presented before (see figure 19), we note that the deficit in streamwise velocity in the wake is on average around $45 \%$, resulting in precisely the observed offset from the VIV peak for an isolated cylinder.

Although we have the evidence to show that vortex-structure interaction is important and necessary for the mechanism to be sustained, so far it is still not clear how vortices from the upstream wake interact with the downstream cylinder during oscillation. Hence, we still need to investigate why vortices enhance the steady and fluctuating lift on the downstream cylinder and also how the phase lag is generated.

\section{Analysis of unsteady lift on an oscillating cylinder}

As discussed before, the WIV response is characterized by considerable variations between cycles as far as displacement is concerned (figure 9); an irregular envelope of displacement is more evident in WIV than in a typical VIV. Figure 23 shows another example of the time series of displacement and lift at $U / D f_{0}=25$, far from the influence of the VIV regime.

In order to investigate the relation between displacement and fluid force in more detail, we shall plot a collection of several superimposed cycles with similar displacement amplitudes. Figure 23 shows the displacement and lift for $20 \%$ of the total number of cycles recorded at this reduced velocity; once more, the variation in both $y$ and $C_{y}$ is evident. It is also clear that the fluid force signal shows a component of higher frequency apart from the lower frequency that matches the cylinder oscillation frequency. Taken as a whole, it appears that the fluid force is indeed out of phase with the displacement; however, if we look carefully at the multitude of light-grey lines crossing $C_{y}=0$ in figure 23 , we will note that the lift force anticipates the displacement practically in all cycles. In fact, if we estimate $\phi$ based on the average cycles given by the black lines, we conclude that the displacement lags the lift by a phase angle which is rather close to the average value of $\phi=161^{\circ}$, calculated as an average of all points represented in the curve ' $\phi$ (Hilbert)' in figure 11. 


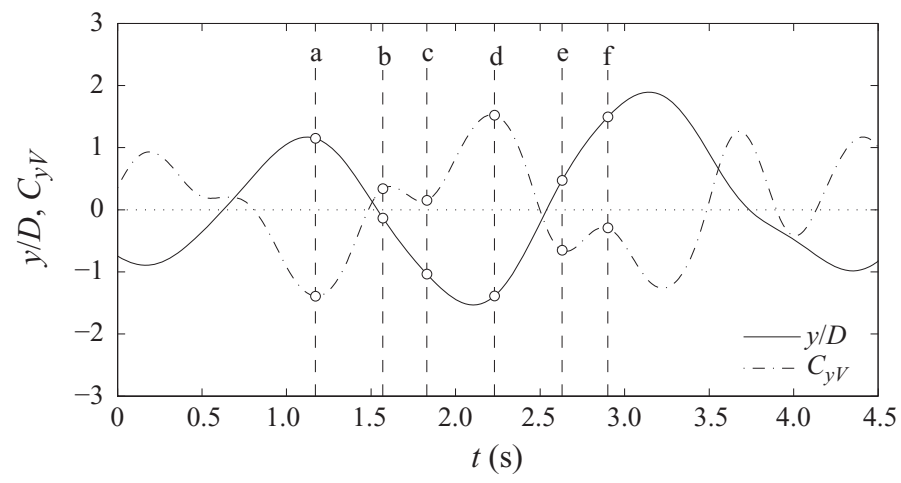

Figure 24. Time series of displacement and vortex-force component of lift on the downstream cylinder under WIV. $x_{0} / D=4.0, U / D f_{0}=25$ and $R e=19200$.

In fact, if we start from (3.1) and take all other variables from the results presented above, we conclude that for such very low values of mass and damping $\left(m^{*} \zeta=0.018\right)$ a minute phase lag of $\phi=179.4$ would be enough to excite the system with $\hat{y} / D=1.5$ at $U / D f_{0}=25$. This is the curve ' $\phi$ (Eq.)' in figure 11 . However, if we employ the same harmonic hypothesis for the actual $\phi=161^{\circ}$ averaged from the Hilbert transform (curve ' $\phi$ (Hilbert)' in figure 11), we conclude that the amplitude of oscillation would reach the unrealistic value of $\hat{y} / D=45$. Hence, we conclude that a simple 'harmonic forcing and harmonic motion' hypothesis does not apply to the WIV mechanism. Rather, a more complex modelling that considers multiple frequencies present in the wake should be developed.

Turning again to figure 23, it appears that the phase lag is accentuated by the existence of the higher frequency present in the lift signal. Since $f_{s}$ from the upstream cylinder is increasing with flow speed as $S t=0.2$ and the oscillation frequency of the downstream cylinder is increasing at a different rate, the relationship between both frequencies is also changing. Essentially, this higher frequency must be associated with the vortex-shedding frequency of the upstream wake - at least this is observed for static cylinders - but one cannot tell how repeatable this forcing is as the downstream cylinder oscillates and interacts with upstream vortices.

The only conclusion we can draw is that with such an irregular forcing it is most likely that the fluid force will not be perfectly in phase (or out of phase) with the displacement, especially as the cylinder crosses the centreline of the wake where strong vortices are present. Therefore, the phase lag must be coming from the unsteadiness of the wake, i.e. from the vortex-structure interaction, as we have been arguing so far.

\subsection{Unsteady vortex-structure interaction}

As in the investigation presented for a static cylinder in a staggered arrangement of $y_{0} / D=1.0$, we can analyse the wake configuration that generates the corresponding lift trace for a cylinder undergoing WIV. Figure 24 shows a short sample from the time series presented in figure 23 for which the flow fields were captured with PIV. Note that the $C_{y_{V}}$ component of lift only accounts for the force generated by the vorticity in the wake. Vorticity contours and velocity fields for six instants 'a' to ' $\mathrm{f}$ ' are shown in figures $25\left(a, a^{\prime}\right)$ to $25\left(f, f^{\prime}\right)$.

Frame 'a' was taken with the cylinder very close to maximum displacement when lift was the strongest towards the centreline. Like the wake configuration observed 

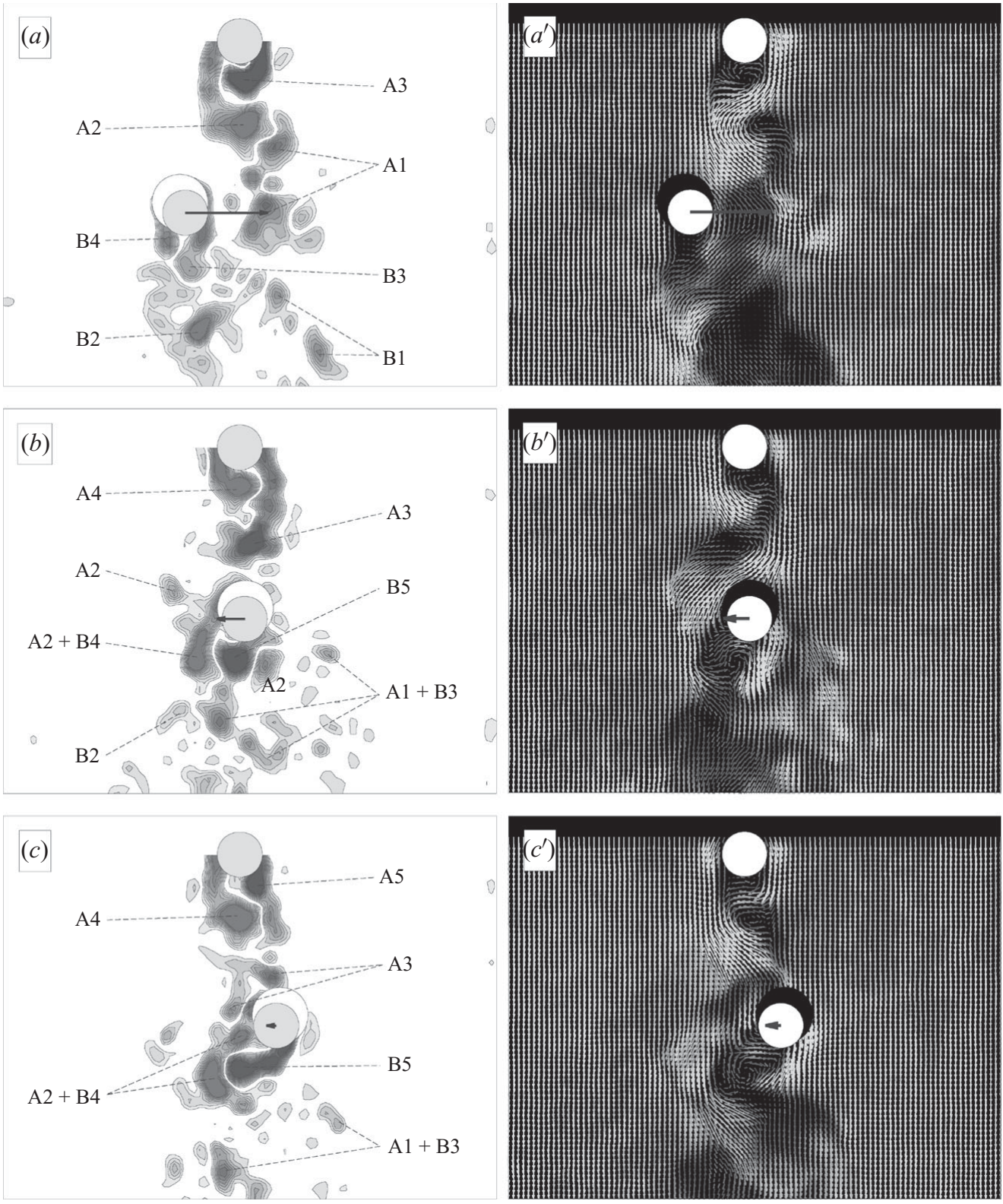

FIGURE 25( $\left.a, a^{\prime}, b, b^{\prime}, c, c^{\prime}\right)$. For caption see facing page.

for static cylinders, we find vortex A2 inducing high-speed flow close to the inner side of the cylinder, while A1 induces vortex B3 to form closer to the cylinder surface. However, as the cylinder accelerates towards the centreline, B3 is suddenly released and a new vortex B5 forms in its place. In figure 24, we see that the lift force changes its direction slightly before the cylinder crosses the centreline. In frame ' $b$ ', we see that vortex A2 impinges on the downstream cylinder, splitting into two parts around the body as it crosses the centreline: one part will merge with B4 and the other part will join B5 in the downstream wake. At the same time, we see that A3 coming from the upstream cylinder induces a high-speed flow that is contrary to the motion of 

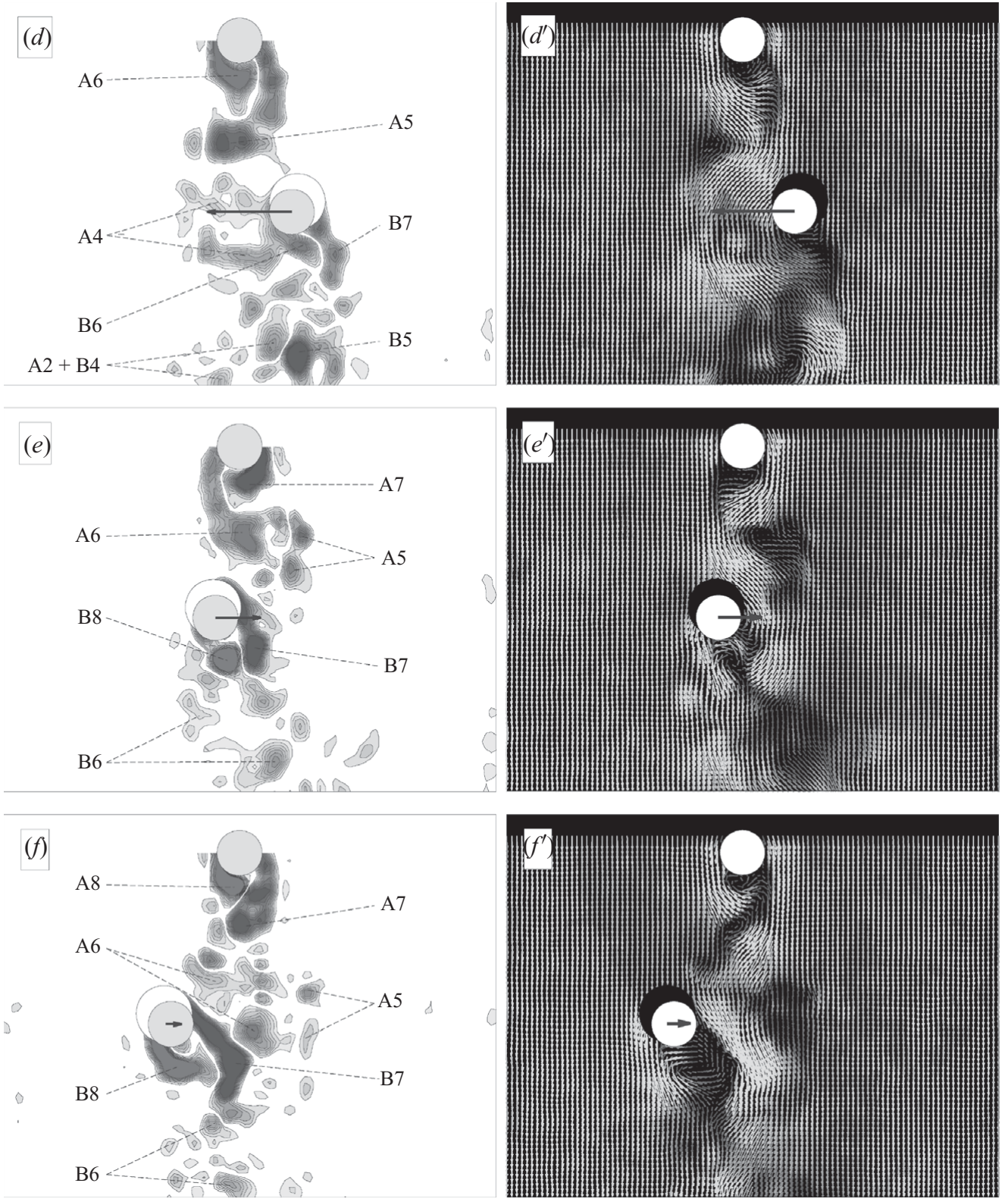

Figure 25( $\left.d, d^{\prime}, e, e^{\prime}, f, f^{\prime}\right) .(a, b, c, d, e, f)$ Instantaneous vorticity contours and $\left(a^{\prime}, b^{\prime}, c^{\prime}, d^{\prime}\right.$, $\left.e^{\prime}, f^{\prime}\right)$ velocity field (coloured by velocity magnitude) obtained with PIV around a pair of cylinder under WIV. Horizontal arrows represent the lift acting on the cylinder (see figure 24). $x_{0} / D=4.0, U / D f_{0}=25$ and $R e=19200$.

the downstream cylinder. This strong interaction is responsible for the local peak in the lift curve in figure 24. A moment later, in frame ' $\mathrm{c}$ ', vortex A3 is splitting around the downstream cylinder and inducing $\mathrm{A} 2+\mathrm{B} 4$ away from the body, while a strong B5 rolls up on the other side resulting in an almost zero lift as the cylinder decelerates towards the minimum peak of response. 
The process is repeated for the other half of the cycle as the cylinder crosses the wake in the opposite direction. Frame 'd' shows that maximum lift towards the centreline occurs when vortex A5 induces high-speed flow on the inner side of the body, while A4 induces B6 to form closer to the cylinder. Note that maximum lift was not registered for the lowermost displacement, but when the cylinder encountered that particular wake configuration on its way towards the centreline. Again, the lift reversal happened slightly before the cylinder crossed the centreline. Similarly, another local peak of lift shown in frame 'e' is caused by the impingement of vortex A5; it splits around the cylinder merging with B7 on the inner side and with B8 on the outer side. Almost zero lift is obtained in frame ' $\mathrm{f}$ ' when the cylinder experiences the same wake interaction as in 'c', though in the opposite direction.

We chose to analyse this portion of the time series because the lift force in the first half of the cycle is mirrored in the second half, giving more of an opportunity to understand the wake-structure interaction taking place. However, we note that the peak of amplitude in frame ' $a$ ' is different from the peak that the cylinder will reach just after frame ' $\mathrm{f}$ '. This reveals that the response of the body is very dependent on the configuration of the wake it encounters for each cycle. With the crossing velocity $\dot{y}$ changing for each cycle, the vortex-structure interaction will also be different, resulting in different responses. In fact, if we look again at the short time series presented in figure 24, we will note that the lift signal for the previous cycle between $t=0$ and $1.0 \mathrm{~s}$ is different from the cycle that we investigated between $t=1.1$ and $3.1 \mathrm{~s}$, and also different from the next cycle after $t=3.1 \mathrm{~s}$. Even the phase lag varies from cycle to cycle. At $t=0.7 \mathrm{~s}$, the opposite is also observed and now it is the displacement that anticipates the fluid force. This variation between successive cycles imposes on the system the irregularity in response observed in the displacement and lift envelopes. But the fact that the phase lag will rarely be equal to zero also guarantees that energy will be transferred from the fluid to the structure sustaining WIV, albeit in irregular amounts from cycle to cycle.

\section{Conclusion}

Up to now, the wake-displacement mechanism proposed by Zdravkovich (1977) seemed to be the most plausible explanation for the WIV phenomenon, even though he could not conclude how the wake was being 'displaced' to generate the necessary phase lag to sustain the vibrations. In this paper, we have shown that WIV is indeed a wake-dependent type of FIV. Yet we found that it is the unsteadiness of the wake that plays the main role in the WIV process and not simply the displacement of a steady flow field.

We suggest that the WIV mechanism is sustained by unsteady vortex-structure interactions that input energy into the system as the downstream cylinder oscillates across the wake.

We have verified that WIV is not a resonant phenomenon. While VIV finds its maximum amplitude of vibration at $f_{s}=f_{0}$, WIV keeps increasing $\hat{y} / D$ even when $f_{s}$ is much higher than $f_{0}$. In the shear-flow experiment, we removed the upstream shedding frequency from the system, leaving only $f_{s}$, which is generated by the downstream cylinder. As a result, the oscillations returned to a typical VIV response, meaning that the upstream frequency - or the upstream vortex shedding was somehow important to sustain the excitation. Hence, WIV is essentially a type of vortex-induced mechanism, in the sense that it requires the interaction of the structure with vortices, even though these vortices are coming from an upstream wake. 
Energy input from the fluid to the structure will only occur when there is a phase lag greater than $\phi=0^{\circ}$ or less than $180^{\circ}$ between the fluid force and displacement. Coherent vortices impinging on the downstream cylinder and merging with its own vortices induce fluctuations in lift that are not synchronized with the motion. Strong vortices from the upstream wake induce considerable changes in the lift force. The shear-flow experiment proved that a steady shear flow without vortices cannot excite a cylinder into WIV. Remove the unsteady vortices from the wake and WIV will not be excited.

The characteristic response of the downstream cylinder is consistent with the arguments presented above. Irregular envelopes of the displacement and lift indicate that the downstream cylinder encounters different wake configurations from cycle to cycle. As the second cylinder is moved farther downstream, vortices coming from the upstream wake have more time to diffuse and the resulting vortex-structure interaction is weakened. This is in agreement with the response curves presented for separations up to $x_{0} / D=20.0$ and also agrees with the fact that the lift force (both steady and fluctuating terms) also diminishes with increasing $x_{0}$. The flow's three-dimensionality is also likely to increase with $x_{0}$ and contribute to reducing the response.

We have shown that WIV had been referred to as a type of galloping mostly because the typical response presents a build-up of amplitude for higher reduced velocities. We argue that quasi-steady assumptions, commonly employed in classical galloping theory, would not fit the WIV phenomenon, as we now understand it. For that reason, we have been insisting on a dissociation of WIV from the classical galloping idea. When Bokaian \& Geoola (1984) call this mechanism 'wake-induced galloping' they are correct in the sense that it is a 1-d.o.f. fluid-elastic mechanism generated by the interference of the upstream wake. However, we do not agree that the excitation mechanism is similar to that of classical galloping of non-circular bluff bodies.

The authors are grateful for the interesting comments made by one of the referees. G.R.S.A. wishes to thank CAPES, Brazilian Ministry of Education, for his $\mathrm{PhD}$ scholarship. J.R.M. thankfully acknowledges Finep-CTPetro/Petrobras, CNPq and Fapesp for financial support. Support from EPSRC is also gratefully acknowledged.

\section{REFERENCES}

Alam, M. M., Moriya, M., Takai, K. \& Sakamoto, H. 2003 Fluctuating fluid forces acting on two circular cylinders in tandem arrangement at a subcritical Reynolds number. J. Wind Engng Ind. Aerodyn. 91, 139-154.

Assi, G. R. S. 2005 Experimental study of the flow interference effect around aligned cylinders. Master's thesis, University of São Paulo, São Paulo, Brazil (in Portuguese). Available at: www.ndf.poli.usp.br/ gassi.

Assi, G. R. S. 2009 Mechanisms for flow-induced vibration of interfering bluff bodies. PhD thesis, Imperial College London, London, UK. Available at: www.ndf.poli.usp.br/ gassi.

Assi, G. R. S., Meneghini, J. R., Aranha, J. A. P., Bearman, P. W. \& Casaprima, E. 2006 Experimental investigation of flow-induced vibration interference between two circular cylinders. J. Fluids Struct. 22, 819-827.

Bearman, P. W. 1984 Vortex shedding from oscillating bluff bodies. Annu. Rev. Fluid Mech. 16, 195-222.

Best, M. S. \& CooK, N. J. 1967 The forces on a circular cylinder in a shear flow. Tech. Rep. 103. Aeronautical Engineering Department, University of Bristol.

Blevins, R. D. 1990 Flow-Induced Vibration, 2nd edn. Van Nostrand Reinhold. 
Bokaian, A. \& Geoola, F. 1984 Wake-induced galloping of two interfering circular cylinders. J. Fluid Mech. 146, 383-415.

BRANKovic, M. 2004 Vortex-induced vibration attenuation of circular cylinders with low mass and damping. PhD thesis, Imperial College, London, UK.

Brika, D. \& LANeville, A. 1999 The flow interaction between a stationary cylinder and a downstream flexible cylinder. J. Fluids Struct. 13, 579-606.

Carmo, B. S., Meneghini, J. R. \& Sherwin, S. J. 2010a Possible states in the flow around two circular cylinders in tandem with separations in the vicinity of the drag inversion spacing. Phys. Fluids 22, 054101.

Carmo, B. S., Meneghini, J. R. \& Sherwin, S. J. $2010 b$ Secondary instabilities in the flow around two circular cylinders in tandem. J. Fluid Mech. 644, 395-431.

Chen, S. S. 1986 A review of flow-induced vibration of two circular cylinders in crossflow. J. Pressure Vessel Tech. 108, 382-393.

Granger, S. \& Paidoussis, M. P. 1996 An improvement to the quasi-steady model with application to cross-flow-induced vibration of tube arrays. J. Fluid Mech. 320, 163-184.

Hahn, S. L. 1996 Hilbert Transforms in Signal Processing. Artech House.

DEN Hartog, J. P. 1956 Mechanical Vibrations, 4th edn. McGraw-Hill.

Hover, F. S. \& Triantafyllou, M. S. 2001 Galloping response of a cylinder with upstream wake interference. J. Fluids Struct. 15, 503-512.

IgARASHI, T. 1981 Characteristics of the flow around two circular cylinders arranged in tandem. Bull. JSME - Japan Soc. Mech. Engng 24, 323-331.

JeSTER, W. \& KallindERIS, Y. 2003 Numerical study of incompressible flow about fixed cylinder pairs. J. Fluids Struct. 17, 561-577.

KhalaK, A. \& Williamson, C. H. K. 1997 Investigation of relative effects of mass and damping in vortex-induced vibration of a circular cylinder. J. Wind Engng. Ind. Aerodyn. 69-71, 341-350.

KhalaK, A. \& Williamson, C. H. K. 1999 Motions, forces and mode transitions in vortex-induced vibrations at low mass-damping. J. Fluids Struct. 13, 813-851.

KING, R. \& Johns, D. J. 1976 Wake interaction experiments with two flexible circular cylinders in flowing water. J. Sound Vib. 45, 259-283.

Lin, J. C., Towfighi, J. \& Rockwell, D. 1995 Instantaneous structure of the near-wake of a circular cylinder: on the effect of Reynolds number. J. Fluids Struct. 9, 409-418.

Lin, J. C., YANG, Y. \& Rockwell, D. 2002 Flow past two cylinders in tandem: instantaneous and average flow structure. J. Fluids Struct. 16, 1059-1071.

LiU, C. H. \& ChEN, J. M. 2002 Observations of hysteresis in flow around two square cylinders in a tandem arrangement. J. Wind Engng Ind. Aerodyn. 90, 1019-1050.

Luungkrona, L., Norberg, C. \& Sunden, B. 1991 Free-stream turbulence and tube spacing effects on surface pressure fluctuations for two tubes in an in-line arrangement. J. Fluids Struct. 5, 701-727.

MaEKaWA, T. 1964 Study on wind pressure against ACSR double conductor. Electr. Engng Japan 84, 21-28.

MaIR, W. A. \& Maull, D. J. 1971 Aerodynamic behaviour of bodies in the wakes of other bodies. Phil. Trans. R. Soc. A 269, 425-437.

Norberg, C. 1998 LDV-measurements in the near wake of a circular cylinder. In Advances in Understanding of Bluff Body Wakes and Flow-Induced Vibration (ed. C. H. K. Williamson \& P. W. Bearman), pp. 1-12. ASME.

Norberg, C. \& Sunden, B. 1987 Turbulence and Reynolds number effects on the flow and fluid forces on a single tube in cross-flow. J. Fluids Struct. 1, 337-357.

Paidoussis, M. P., Mavriplis, D. \& Price, S. J. 1984 A potential-flow theory for the dynamics of cylinder arrays in cross-flow. J. Fluid Mech. 146, 227-252.

Paidoussis, M. P. \& Price, S. J. 1988 The mechanisms underlying flow-induced instabilities of cylinder arrays in crossflow. J. Fluid Mech. 187, 45-59.

Papaioannou, G., Yue, D. K. P, Triantafyllou, M. S. \& Karniadakis, G. E. 2006 Threedimensionality effects in flow around two tandem cylinders. J. Fluid Mech. 558, 387-413.

Parkinson, G. V. 1971 Wind-induced instability of structures. Phil. Trans. R. Soc. Lond. 269, 395-413.

Parkinson, G. V. 1989 Phenomena and modelling of flow-induced vibrations of bluff bodies. Prog. Aerosp. Sci. 26, 169-224. 
Price, S. J. 1976 The origin and nature of the lift force on the leeward of two bluff bodies. Aeronaut. Q. 26, 1154-1168.

Price, S. J. 1984 An improved mathematical model for the stability of cylinder rows subject to cross-flow. J. Sound Vib. 97, 615-640.

Sarpkaya, T. 1979 Vortex-induced oscillations: a selective review. J. Appl. Mech. 46, 241-258.

SARPKAYA, T. 2004 A critical review of the intrinsic nature of vortex-induced vibrations. J. Fluids Struct. 19, 389-447.

Sumner, D., Price, S. J. \& Paidoussis, M. P. 2000 Flow-pattern identification for two staggered circular cylinders in cross-flow. J. Fluid Mech. 411, 263-303.

WardLAW, R. L. \& WATTS, J. A. 1974 Wind-tunnel and analytical investigations into the aeroelastic behaviour of bundled conductors. In IEEE Power Engineering Society Summer Meeting, Anahein, CA.

Williamson, C. H. K. \& Govardhan, R. 2004 Vortex-induced vibrations. Annu. Rev. Fluid Mech. 36, 413-455.

ZDRAVkovich, M. M. 1974 Flow-induced vibrations of two cylinders in tandem and their suppression. In International Symposium of Flow Induced Structural Vibrations (ed. E. Naudascher), pp. 631-639. Springer, Berlin.

ZDRavkovich, M. M. 1977 Review of flow interference between two circular cylinders in various arrangements. J. Fluids Engng pp. 618-633.

Zdravkovich, M. M. 1985 Flow induced oscillations of two interfering circular cylinders. J. Sound Vib. 101, 511-521.

ZDRavkovich, M. M. 1986 Discussion: effect of vibrating upstream cylinder of two circular cylinders in tandem arrangement. J. Fluids Engng 108 (3), 383-384.

ZdRavkovich, M. M. 1988 Review of interference-induced oscillations in flow past two circular cylinders in various arrangements. J. Wind Engng Ind. Aerodyn. 28, 183-200.

Zdravkovich, M. M. \& Medeiros, E. B. 1991 Effect of damping on interference-induced oscillations of two identical circular cylinders. J. Wind Engng Ind. Aerodyn. 38, 197-211. 